\title{
Real-time dynamics in diluted quantum networks
}

\author{
Erik Aurell $\odot,{ }^{1, *}$ Roberto Mulet, ${ }^{2, \dagger}$ and Jan Tuziemski ${ }^{3,4,5, \$}$ \\ ${ }^{1}$ KTH-Royal Institute of Technology, AlbaNova University Center, SE-106 91 Stockholm, Sweden \\ ${ }^{2}$ Group of Complex Systems and Statistical Physics, Department of Theoretical Physics, Physics Faculty, University of Havana, Cuba \\ ${ }^{3}$ Department of Physics, Stockholm University, AlbaNova University Center, Stockholm SE-106 91 Sweden \\ ${ }^{4}$ Nordita, Royal Institute of Technology and Stockholm University, Roslagstullsbacken 23, SE-106 91 Stockholm, Sweden \\ ${ }^{5}$ Department of Applied Physics and Mathematics, Gdańsk University of Technology, 80-233 Gdańsk, Poland
}

(Received 10 August 2021; revised 5 October 2021; accepted 7 January 2022; published 9 February 2022)

\begin{abstract}
We introduce an approach to characterize the dynamics of disordered quantum networks. Each quantum element (i.e., each node) of the network experiences the other nodes as an effective environment that can be self-consistently represented by a Feynman-Vernon influence functional. For networks having the topology of locally treelike graphs, these Feynman-Vernon (FV) functionals can be determined by a new version of the cavity or belief propagation (BP) method. Here, we find the fixed point solution of this version of BP for a network of uniform quantum harmonic oscillators. Then, we estimate the effects of the disorder in these networks within the replica symmetry ansatz. We show that over a large time interval, at small disorder, the real part of the FV functional induces decoherence and classicality while at sufficiently large disorder the Feynman-Vernon functional tends to zero and the coherence survives, signaling in a time setting, the onset of an Anderson's transition.
\end{abstract}

DOI: 10.1103/PhysRevA.105.022205

\section{INTRODUCTION}

This work proposes an approach to describe real-time dynamics of quantum networks. The approach is geared toward the evolution of single systems (nodes) in networks of locally treelike topology, such as random graphs or random hypergraphs. As we will see, there is a natural dichotomy between networks without disorder, where grosso modo each system in the network behaves as if interacting strongly with a bath, and networks with disorder. In the latter, each node experiences the network as dissipation, but shielded from decoherence, implying the presence of a different mechanism to preserve quantumness that is independent of the single constituents of the network.

To begin explaining the approach it is convenient to bring in the classical cavity method; a way to simultaneously compute all marginals of a Gibbs-Boltzmann distribution when the interaction graph has no short loops. It is computationally efficient, taking a time polynomial in system size, exact when interactions form tree graphs, and in many cases

\footnotetext{
*eaurell@kth.se

†roberto.mulet@gmail.com

${ }^{\ddagger}$ On leave from Gdańsk University of Technology; jan.tuziemski@fysik.su.se
}

Published by the American Physical Society under the terms of the Creative Commons Attribution 4.0 International license. Further distribution of this work must maintain attribution to the author(s) and the published article's title, journal citation, and DOI. Funded by Bibsam. asymptotically exact when the graph size tends to infinity. Comprehensive modern references are Refs. [1-3]. The cavity method has found many applications to biology, sociology, computer science and artificial intelligence, where it is often called belief propagation (BP) [4].

A version of the cavity method for quantum systems was introduced in 1973 [5]. In that pioneering version, the object is the time-independent wave function on a Bethe lattice or a Cayley tree; cavity equations connect different sites where energy enters as a parameter. Recent applications of this family of methods have been to bosons on the Cayley tree [6], and to the Anderson transition on random graphs [7,8]. A second type of applications of the cavity method to quantum problems is to thermal equilibrium states $[9,10]$. Several other attempts have been made to generalize BP to quantum systems by taking BP messages to be operator-valued. A selection of papers in this direction and in the ones outlined above are Refs. [11-17].

Our approach is an extension of the cavity method to study the real-time dynamics of quantum systems. We exploit the influence functional technique developed by Feynman and Vernon [18] quite long ago, and we show that the real-time quantum cavity can be realized as transformations of influence functionals on influence functionals. While for Feynman and Vernon the goal was to understand the influence of an environment on a test system, in this work the system of interest is the environment or network itself. We want to understand the dynamical behavior of many quantum interacting units influencing each other.

We exploit the essence of the cavity method, the marginalization of the variables of interest, starting - in this casefrom the reduced density matrix of an open system. However, 
in contrast to quantum cavity for thermal equilibrium states, each node of the network is in the real-time dynamics described by two histories and two Feynman path integrals. The result of integrating out all the other histories is an influence functional acting on one node only [18]. The difficulty is then, to calculate self-consistently all these functionals. The main goal of this paper is to show how to make this computation but also to present their properties and consequences, in simple but still relevant cases.

Some of the current computing architectures are based on units with limited connectivity, such as 27-qubit Falcon and 5-qubit Canary processors designed by IBM [19]. Moreover, the properties of networks of such systems have been investigated extensively [20-24], e.g., in the context of generation arbitrary quantum states using local operations and shared entanglement between the nodes [20]. In addition, a platform to simulate quatum Cayley-tree Ising Hamiltonians was realized using Rydberg atoms [25]. The real-time quantum cavity method introduced here gives a different approach to describe the dissipation and decoherence present in such setups. This may prove useful for developing noise characterization and mitigation techniques for current noisy intermediate scale quantum (NISQ) devices [26] but also for the developing of novel results regarding noise threshold theorems for faulttolerant quantum computation [27].

The rest of this manuscript is organized as follows: In Sec. II we briefly introduce the cavity method in its general form. Although a well-established technique in statistical physics, it is less known in quantum science and quantum information, and we hence offer this summary for readers of this community. After this introduction, we show in Sec. III how these ideas can be extended to study quantum dynamics in diluted networks. Then, in Sec. IV we present exact results for networks of uniform quantum harmonic oscillators, and in Sec. V we loosen the requirement of uniformity and extend the analysis - at the replica symmetric level-to networks with quenched disorder. Finally, we present the conclusions and an outlook of our work Sec. VI. Technical details are given in a series of Appendices.

\section{INTRODUCTION TO THE CAVITY METHOD}

The cavity method in its most widely used incarnation is a way to simultaneously compute all marginals of a GibbsBoltzmann distribution when the interaction graph has no short loops [1-3]. The interaction graph is here a representation of an energy function containing only pairwise terms: there is a link $i$ - $j$ between units $i$ and $j$ if there is term in the energy function which depends on the corresponding variables $X_{i}$ and $X_{j}$. The cavity method can be straightforwardly generalized to energy functions containing more than pairwise interactions which are then represented as hypergraphs. Such systems can however always be reduced to pairwise interactions by introducing appropriate dummy variables and constraints.

The starting point of the cavity method, still of this most common type, is a joint probability distributions of the type

$$
P\left(X_{1}, \ldots, X_{N}\right)=\frac{1}{Z} \prod_{i} \phi_{i}\left(X_{i}\right) \prod_{i j} \psi_{i j}\left(X_{i}, X_{j}\right),
$$

where $\phi_{i}\left(X_{i}\right)$ are one-body terms, $\psi_{i j}\left(X_{i}, X_{j}\right)$ are two-body terms, and $Z$ is a normalization constant. For GibbsBoltzmann distributions $\phi_{i}\left(X_{i}\right)=e^{-\beta E_{i}\left(X_{i}\right)}$ and $\psi_{i j}\left(X_{i}, X_{j}\right)=$ $e^{-\beta E_{i j}\left(X_{i}, X_{j}\right)}, \quad \beta=\frac{1}{k_{B} T}$ and $T$ is temperature and $k_{B}$ is Boltzmann's constant. Equation (1) is however more general; $\psi_{i j}\left(X_{i}, X_{j}\right)$ can for instance encode hard constraints which do not depend on temperature. In the next section we will be concerned with a yet wider generalization, where $P\left(X_{1}, \ldots, X_{N}\right)$ is a multivariable quantum mechanical path probability amplitude (of both forward and backward paths), and $Z$ is implicit in the normalization of Feynman path integrals.

In classical systems, the cavity method is from a formal point of view embodied in BP output equations and BP update equations. We illustrate these two types of equations in Fig 1. The BP output equations are

$$
p_{i}\left(X_{i}\right)=\frac{1}{Z_{i}} \phi\left(X_{i}\right) \prod_{a \in \partial i} m_{a \rightarrow i}\left(X_{i}\right), \quad a=(i-j),
$$

where $p_{i}\left(X_{i}\right)$ is the BP approximation to the marginal probability of variable $X_{i}, Z_{i}$ is a normalization, and $m_{a \rightarrow i}\left(X_{i}\right)$ is the contribution to the marginal distribution from "summing out" all variables in the graph starting from neigbor $j$ of $i$. The use of $a$ to denote the interaction term $i$ - $j$ is conventional; the second product in above could therefore also have been written $\prod_{j \in \partial i} m_{(i j) \rightarrow i}\left(X_{i}\right)$.

In our generalization $p_{i}\left(X_{i}, Y_{i}\right)$, which has to depend on two variables of the same type, is the probability amplitude of system $i$, and $m_{a \rightarrow i}\left(X_{i}, Y_{i}\right)$ are the Feynman-Vernon influence functionals from integrating out all variable in the network starting from $a$. Note that the preceding sentence has a precise meaning if the network is a tree. If in fact the network is only locally treelike, then $m_{a \rightarrow i}\left(X_{i}, Y_{i}\right)$ should be looked at as a part of an approximation to a total Feynman-Vernon influence functional as discussed below.

The BP update equations for classical probability distributions are a pair of equations

$$
\begin{gathered}
n_{i \rightarrow a}\left(X_{i}\right)=\frac{1}{\Omega_{i \rightarrow a}} \phi\left(X_{i}\right) \prod_{b \in \partial i \backslash a} m_{b \rightarrow i}\left(X_{i}\right), \\
m_{a \rightarrow i}\left(X_{i}\right)=\frac{1}{\Omega_{a \rightarrow i}} \sum_{X_{a} \backslash X_{i}} \psi_{a}\left(X_{a}\right) \prod_{j \in \partial a \backslash i} n_{j \rightarrow a}\left(X_{j}\right),
\end{gathered}
$$

where $\Omega_{i \rightarrow a}$ and $\Omega_{a \rightarrow i}$ are normalization constants and $X_{a}$ is a shorthand for the pair $\left(X_{i}, X_{j}\right)$ [the sum $\sum_{X_{a} \backslash X_{i}}$ is thus over $X_{j}$ and the product $\prod_{j \in \partial a \backslash i} n_{j \rightarrow a}\left(X_{j}\right)$ contains only one term; as written the expressions however also hold for multivariable interactions]. In our generalization the sum $\sum_{X_{a} \backslash X_{i}}$ goes over to $\sum_{\left(X_{a}, Y_{a}\right) \backslash\left(X_{i}, Y_{i}\right)}$ which is a (multivariable) path integral, and the normalizations $\Omega_{i \rightarrow a}$ and $\Omega_{a \rightarrow i}$ are implicit in these path integrals.

In the real-time quantum cavity method which we introduce $m_{j \rightarrow i}$ and $n_{j \rightarrow i}$ are very high-dimensional objects, and the update step ( $n$ to $m$ iteration) is therefore computationally expensive. For harmonic networks this aspect is mitigated as one can then use the Feynman-Vernon theory with closedform expressions for the actions in the influence functionals.

A useful analogy for the more general computational challenge is the cavity method applied to describe the dynamics 

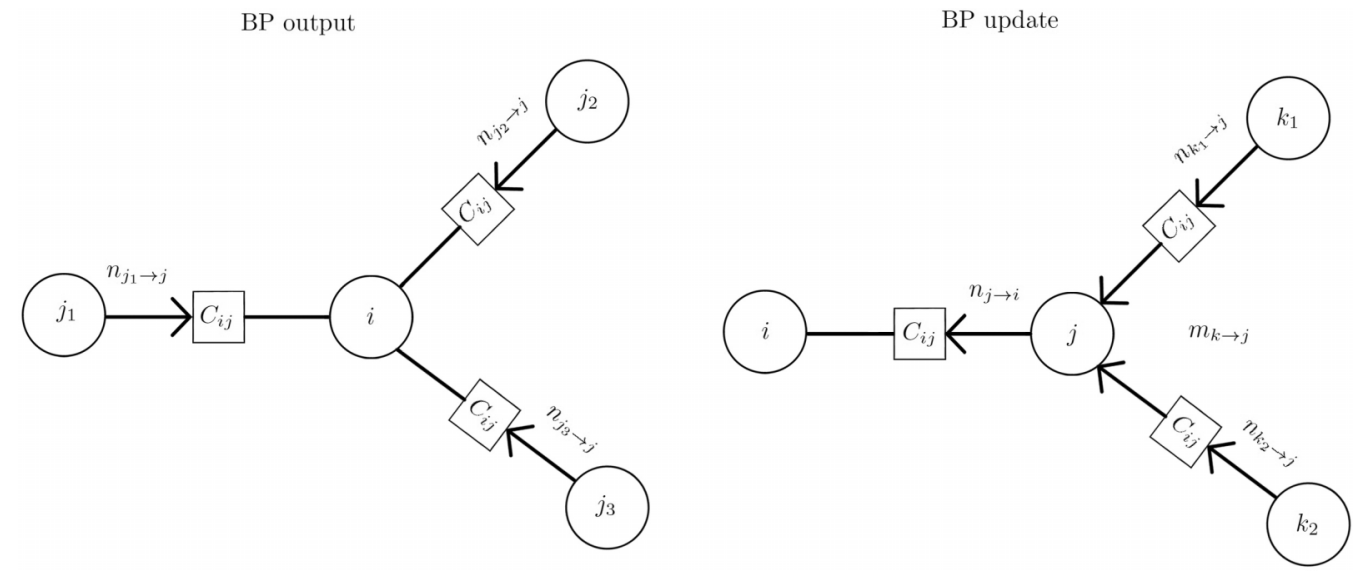

FIG. 1. Left panel: illustration of the marginal probability amplitude over local histories as expressed by Eq. (2). In the real-time cavity method messages $n_{j \rightarrow i}$ are equal to $\exp \left(\frac{i}{\hbar} F_{j \rightarrow i}\right)$ where $F_{j \rightarrow i}$ is a Feynman-Vernon action discussed in text representing the effect of integrating out nodes in the graph subtended from $j$, but not $j$ itself. Right panel: illustration of the recursive Eqs. (3) and (4). Nodes labeled $k$ are here coupled to a node labeled $j$, which in turn is coupled to a node labeled $i$. Message $m_{j \rightarrow i}$ is obtained by a double path integral over the histories $X_{j}$ and $Y_{j}$ of node $j$ with the bare action $S_{j}$, the interaction action $S_{i j}$, and the upstream influence $F_{j \rightarrow i}$. This message hence represents integrating out all nodes subtended from node $j$, and node $j$ itself. Message $n_{j \rightarrow i}$ is on the hand obtained by combining messages $m_{k \rightarrow j}$ for $k$ in the neighborhood of $j$ except $i$.

of classical spin systems [28-39]. Also in this case $m_{j \rightarrow i}$ and $n_{j \rightarrow i}$ are very high-dimensional objects, and the update step ( $n$ to $m$ iteration) computationally expensive. Further assumptions or approximations are therefore also then needed to get useful results; in Ref. [28] it is assumed that interactions are fully asymmetric, which allows for marginalization over time; $m_{j \rightarrow i}$ and $n_{j \rightarrow i}$ then become low-dimensional objects. Alternatively one can do Markov closure in discrete or continuous time [32,35-37] to reduce the dynamics to low-dimensional iterations, the accuracy of which however needs to be assessed numerically. More advanced approximations have also been explored, in both discrete and continuous time [33,34,39].

\section{THE REAL-TIME QUANTUM CAVITY}

We now proceed to define BP for the dynamics of quantum networks interacting as locally treelike graphs. Each system $i$ in the network is represented by a set of commuting observables which we write $\hat{X}_{i}$, possibly with several components. The corresponding time-dependent coordinate integrated over in the path integral approach is written $X_{i}(t)$. The variables which we start from are the trajectories $X_{1} \ldots X_{N}$ of the degrees of freedom of the systems in the network, and the state of the network is the full density matrix, $\rho(t)=U(t) \rho_{0} U^{\dagger}(t)$. In the path integral approach this is expressed as

$$
\begin{aligned}
U(t) & \cdot U^{\dagger}(t) \\
= & \int D X_{i} D Y_{i} \cdots \exp \frac{i}{\hbar}\left(S\left[X_{i}\right]+S\left[X_{j}\right]-S\left[Y_{i}\right]\right. \\
& \left.-S\left[Y_{j}\right]+S\left[X_{i}, X_{j}\right]-S\left[Y_{i}, Y_{j}\right]+\cdots\right) \\
& \times \rho_{0}\left[x_{i}\left(t_{i}\right), \ldots, y_{i}\left(t_{i}\right), \ldots\right]
\end{aligned}
$$

where $\rho_{0}\left[x_{i}\left(t_{i}\right), \ldots, y_{i}\left(t_{i}\right), \ldots\right]$ is the initial density operator of the whole network. A characteristic feature of real-time quantum dynamics is that for each system there is one "forward path" (denoted $X$, representing $U$ ) and one "backward path" $\left(Y\right.$, representing $\left.U^{\dagger}\right)$ [18]. The action contains two kind of constitutive action parts. $S\left[X_{i}\right]$ and $S\left[Y_{i}\right]$ are the selfinteractions of system $i$ and thus represent the evolution of the density matrix by a single-system Hamiltonian $H_{i} . S\left[X_{i}, X_{j}\right]$ and $S\left[Y_{i}, Y_{j}\right]$ are the interactions between systems $i$ and $j$, neighbors in the interaction graph, and represent the evolution of the density matrix by a system-system interaction Hamiltonian $H_{i j}$.

We are interested in the reduced dynamics of the $i$ th node, obtained by tracing out all other nodes. Tracing first out all units except the $i$ th unit and its neighbors labeled $j$, we can rewrite Eq. (5) as an evolution equation for the density matrix of system or node $i$ :

$$
\begin{aligned}
\rho_{f}^{(i)}\left[X_{i}\left(t_{f}\right), Y_{i}\left(t_{f}\right)\right] \\
=\int D X_{i} D Y_{i} \prod_{j \in \partial i} D X_{j} D Y_{j} \\
\quad \times \exp \left[\frac { i } { \hbar } \left(S\left[X_{i}\right]-S\left[Y_{i}\right]+S\left[X_{j}\right]\right.\right. \\
\left.\left.\quad-S\left[Y_{j}\right]+S\left[X_{i}, X_{j}\right]-S\left[Y_{i}, Y_{j}\right]\right)\right] \\
\quad \times \exp \left\{\frac{i}{\hbar} \mathcal{F}_{\partial i}\left[\left(X_{j}, Y_{j}\right)_{j \in \partial i}\right]\right\} \rho_{0}^{(i)}\left[x_{i}\left(t_{i}\right), y_{i}\left(t_{i}\right)\right] \\
\quad \times \prod_{j \in \partial i} \delta\left[x_{j}\left(t_{f}\right)-y_{j}\left(t_{f}\right)\right] \rho_{0}^{(j)}\left[x_{j}\left(t_{i}\right), y_{j}\left(t_{i}\right)\right] .
\end{aligned}
$$

Here and in the following we assume a factorized initial state, and we explicitly trace the final state of all states $j \in \partial i$, the set of neighbors of $i$. The functional $\mathcal{F}_{\partial i}$ is the result of integrating over the histories and tracing the final state of all variables except those in node $i$ and $\partial i$. It is a functional of the histories in $\partial i$, but does not know about $i$ itself. In cavity method language one says that variable $i$ has been removed, and its place in the original network has been replaced by a cavity. 
The locally treelike geometry means that the variables in $\partial i$ are far apart in this new cavity network. They are not independent, but after $i$ has been removed their dependence is through many intermediate nodes. The fundamental assumption of the cavity method on the replica symmetric (RS) level is that in a large enough network the nodes in $\partial i$ are eventually independent. This means that $\mathcal{F}_{\partial i}$ simplifies as

$$
\mathcal{F}_{\partial i}=\sum_{j \in \partial i} F_{j \rightarrow i}\left[X_{j}, Y_{j}\right] \quad(\text { RS cavity assumption })
$$

Structurally, Eq. (6) with Eq. (7) is now a BP output equation where the the $F_{j \rightarrow i}$ play the roles of BP messages.

To put this into practical terms, this real-time quantum cavity method exploits the Feynman-Vernon functionals integrating out a whole tree subtended by one node. As discussed in the previous section these are of two types, conventionally in the cavity literature called " $n$-type" and and " $m$-type" messages. When all terms in the action are quadratic we parametrize these messages as explicit Feynman-Vernon actions [18]:

$$
\begin{aligned}
n_{j \rightarrow i}\left[X_{j}, Y_{j}\right]=e^{\frac{i}{\hbar} F_{j \rightarrow i}\left[X_{j}, Y_{j}\right]}= & \exp \left\{\frac{i}{\hbar} \int_{\tau}^{T} \int_{\tau}^{t} k_{I}^{j \rightarrow i}(s, t-s)\left[X_{j}(t)-Y_{j}(t)\right]\left[X_{j}(s)+Y_{j}(s)\right] d t d s\right\} \\
& \times \exp \left\{-\frac{1}{\hbar} \int_{\tau}^{T} \int_{\tau}^{t} k_{R}^{j \rightarrow i}(t, s)\left[X_{j}(t)-Y_{j}(t)\right]\left[X_{j}(s)-Y_{j}(s)\right] d t d s\right\}, \\
m_{j \rightarrow i}\left[X_{i}, Y_{i}\right]=e^{\frac{i}{\hbar} \tilde{F}_{j \rightarrow i}\left[X_{i}, Y_{i}\right]}= & \exp \left\{\frac{i}{\hbar} \int_{\tau}^{T} \int_{\tau}^{t} \tilde{k}_{I}^{j \rightarrow i}(s, t-s)\left[X_{i}(t)-Y_{i}(t)\right]\left[X_{i}(s)+Y_{i}(s)\right] d t d s\right\} \\
& \times \exp \left\{-\frac{1}{\hbar} \int_{\tau}^{T} \int_{\tau}^{t} \tilde{k}_{R}^{j \rightarrow i}(t, s)\left[X_{i}(t)-Y_{i}(t)\right]\left[X_{i}(s)-Y_{i}(s)\right] d t d s\right\} .
\end{aligned}
$$

The kernels $k^{j \rightarrow i}$ (symbol without tilde) in $n_{j \rightarrow i}$ multiply histories pertaining to the ingress node (node $j$ ). They represent the effect of integrating out the histories of the systems in all nodes neighbors to $j$ or subtended from neighbors of $j$, except node $i$ and nodes subtended from $i$. If the network is not harmonic, then the Feynman-Vernon action contains terms of every order (cubic, quartic, etc.). The kernels of those higher order terms are given by connected correlation functions in the environment, in analogy to that $k_{I}$ and $k_{R}$ in above are related to the pairwise correlation functions in a harmonic environment [40].

One relation between $n$-messages and $m$-messages follows from the most basic property of influence functionals; that influence functions from disjoint environments multiply. In our case we write this as

$$
n_{j \rightarrow i}\left[X_{j}, Y_{j}\right] \equiv \exp \left[\frac{i}{\hbar} F_{j \rightarrow i}\left[X_{j}, Y_{j}\right]\right]=\prod_{k \in \partial j \backslash i} m_{k \rightarrow j}\left[X_{j}, Y_{j}\right] \equiv \exp \left[\frac{i}{\hbar} \sum_{k \in \partial j \backslash i} \tilde{F}_{k \rightarrow j}\left[X_{j}, Y_{j}\right]\right],
$$

which for the kernels translate to

$$
\begin{aligned}
& k_{I}^{j \rightarrow i}(s, t-s)=\sum_{k \in \partial j \backslash i} \tilde{k}_{I}^{k \rightarrow j}(s, t-s), \\
& k_{R}^{j \rightarrow i}(s, t-s)=\sum_{k \in \partial j \backslash i} \tilde{k}_{R}^{k \rightarrow j}(s, t-s) .
\end{aligned}
$$

This relation is the same also for nonharmonic networks; the kernels of the higher-order Feynman-Vernon action terms sum in the same way.

The other relation between $n$ messages and $m$ messages follows from integrating out the histories of the system in node $j$. In general we can formally write this mapping as

$$
\begin{aligned}
m_{k \rightarrow j}\left[X_{j}, Y_{j}\right] \equiv & \int D X_{k} D Y_{k} \exp \left[\frac{i}{\hbar}\left(S\left[X_{k}\right]-S\left[Y_{k}\right]+S\left[X_{j}, X_{k}\right]-S\left[Y_{j}, Y_{k}\right]\right)\right] \\
& \times n_{k \rightarrow j}\left[X_{k}, Y_{k}\right] \delta\left[x_{k}\left(t_{f}\right)-y_{k}\left(t_{f}\right)\right] \rho_{0}^{(k)}\left[x_{k}\left(t_{i}\right), y_{k}\left(t_{i}\right)\right] .
\end{aligned}
$$

This is the step which is computationally costly and which in general cannot be done without further assumptions. The case of harmonic degrees of freedom is considered in the following section.

\section{QUANTUM HARMONIC OSCILLATORS}

As a first solvable example with unexpected properties, we now discuss a uniform random network of harmonic oscillators which interact linearly. The action is then $\mathcal{S}\left[X_{1}, \cdots, X_{N}\right]=\int_{t_{i}}^{t_{f}} \frac{m}{2} \sum_{j} \dot{X}_{j}^{2}-\frac{m \omega_{0, j}^{2}}{2} X_{j}^{2}+\frac{1}{2} \sum_{j, i \in \partial j} C_{i j}\left(X_{i}-X_{j}\right)^{2} d t$, where $m$ is the oscillator mass, $\omega_{0, j}$ is the frequency, and $C_{i j}$ is the spring constant between oscillator $i$ and $j$. The single-system action parts are $S\left[X_{j}\right]=\int_{t_{i}}^{t_{f}} \frac{m}{2} \dot{X}_{j}^{2}-\frac{m \omega_{0}^{2}+\sum_{i \in \partial j} C_{i j}}{2} X_{j}^{2} d t$, and it is convenient to use the notation $m \omega^{2}=m \omega_{0}^{2}+\sum_{i \in \partial j} C_{i j}$. The system-system actions are $S\left[X_{j}, X_{k}\right]=\int_{t_{i}}^{t_{f}}\left(-C_{i j}\right) X_{j} X_{k} d t$. All terms in the action are quadratic, and one could suppose the problem solvable by diagonalization. However, the initial conditions have been assumed factorized over the nodes in the network which is a 
condition on the original coordinates, not on the modes obtained after diagonalization. To solve the problem is therefore not trivial. Additionally, except in one dimension the total Hamiltonian is partly random only from the structure of the locally treelike graph.

For the harmonic oscillator in node $j$, under the action of the interactions discussed above the Feynman-Vernon path integral to consider is

$$
\begin{aligned}
e^{\frac{i}{\hbar} \tilde{F}_{j \rightarrow i}\left[X_{i}, Y_{i}\right]}= & \int \exp \left\{\frac{i}{\hbar} \int_{\tau}^{T}\left[\frac{m}{2}\left(\dot{X}_{j}^{2}-\dot{Y}_{j}^{2}\right)-\frac{m \omega^{2}}{2}\left(X_{j}^{2}-Y_{j}^{2}\right)+C_{i j} X_{i} X_{j}-C_{i j} Y_{i} Y_{j}\right] d t\right\} e^{\frac{i}{\hbar} F_{j \rightarrow i}\left[X_{j}, Y_{j}\right]} \\
& \delta\left[X_{j}(T)-Y_{j}(T)\right] \frac{1}{N} \exp \left[-\left(A x^{2}+2 B x y+C y^{2}\right) / 2\right] \mathcal{D} X_{j} \mathcal{D} Y_{j} d X_{j}(\tau) d Y_{j}(\tau) d X_{j}(T) d Y_{j}(T) .
\end{aligned}
$$

Lowercase letters $(x$ and $y)$ in the above stand for the initial data on $j$, i.e., $X_{j}(\tau)$ and $Y_{j}(\tau)$, and uppercase letters $(X$ and $Y)$ will from now on stand for the final data $X_{j}(T)$ and $Y_{j}(T)$. The core problem is to translate Eq. (14) into a transformation of kernels, while the geometry of passing of message of types $m$ and $n$ remains as in Fig. 1.

It turns out that Eq. (14) is a kind of iterated path integral first considered by Vernon in Appendix V of his Ph.D. thesis [41] and which we call the Vernon transform. The transform has been reinvented at least once in the more recent literature [42]. We can therefore immediately write down

$$
\tilde{k}_{I}^{j \rightarrow i}=\mathcal{V}\left[k_{I}^{j \rightarrow i}, C_{i j}\right]=\frac{1}{2} C_{i j}(t) C_{i j}(s) G^{j \rightarrow i}(t, s-t),
$$

where $G^{j \rightarrow i}$ is a response function described below. Neglecting the contribution from the initial condition of oscillator $j$ (see Appendix A for details) the real side of the Vernon transform $\mathcal{W}$ is

$$
\tilde{k}_{R}^{j \rightarrow i}(t, s)=\mathcal{W}\left[k_{R}^{j \rightarrow i}, C_{i j}\right]=C_{i j}(t) C_{i j}(s) \int_{\tau}^{t} \int_{\tau}^{s} k_{R}^{j \rightarrow i}\left(t^{\prime}, s^{\prime}\right) G^{j \rightarrow i}\left(t^{\prime}, t-t^{\prime}\right) G^{j \rightarrow i}\left(s^{\prime}, s-s^{\prime}\right) d s^{\prime} d t^{\prime},
$$

with the same response function $G^{j \rightarrow i}$ as in Eq. (A11).

Equations (15) and (16) can be closed through Eq. (10) as

$$
\begin{aligned}
k_{I}^{j \rightarrow i}(t, s-t) & =\sum_{k \in \partial j / i} \tilde{k}_{I}^{k \rightarrow j}(t, s-t)=\sum_{k \in \partial j / i} \frac{1}{2} C_{k j}(t) C_{k j}(s) G(t, s-t), \\
k_{R}^{j \rightarrow i}(t, s) & =\sum_{k \in \partial j / i} \tilde{k}_{R}^{k \rightarrow j}(t, s)=\sum_{k \in \partial j / i} C_{k j}(t) C_{k j}(s) \int_{-\infty}^{t} \int_{-\infty}^{s} k_{R}^{k \rightarrow j}\left(t^{\prime}, s^{\prime}\right) G\left(t^{\prime}, t-t^{\prime}\right) G\left(s^{\prime}, s-s^{\prime}\right) d s^{\prime} d t^{\prime} .
\end{aligned}
$$

The information about the graph structure is given through the introduction of the $\sum_{k \in \partial j / i}$. Equations (17) constitute the BP update equations of the problem and constitute integral transformations on the Feynman-Vernon kernels.

However (for derivations, see Appendices A and B), the response function and the friction kernel satisfy the twinning relation

$$
G^{j \rightarrow i}(t, s-t)=G_{j}^{(0)}(s-t)+\int_{t}^{T} d t_{1} \int_{t_{1}}^{T} d t_{2} G_{j}^{(0)}\left(t_{1}-t\right) \quad k_{I}^{k \rightarrow j}\left(t_{1}, t_{2}-t_{1}\right) G^{j \rightarrow i}\left(t_{2}, s-t_{2}\right),
$$

which, under the assumption of independence from the first time argument, in the Laplace domain becomes

$$
\tilde{G}(\lambda)=\tilde{G}_{0}(\lambda)+\tilde{G}_{0}(\lambda) k_{I}^{k \rightarrow j}(\lambda) \tilde{G}(\lambda),
$$

where for simplicity we used the same symbol for $k_{I}^{k \rightarrow j}$ in the time domain and in the Laplace domain and dropped the supraindexes. The Laplace transform $\tilde{G}_{0}(\lambda)$ is given by

$$
\tilde{G}_{0}(\lambda)=\int_{0}^{\infty} e^{-\lambda t} \frac{2}{m \omega} \sin \omega t d t=\frac{2}{m} \frac{1}{\lambda^{2}+\omega^{2}} .
$$

This is twice the response function of the harmonic oscillator as conventionally defined.

In other words, when the process has been going on for a long time under constant conditions the transform $\mathcal{V}$ of $k_{I}$ simplifies greatly on the Laplace or Fourier transform side:

$$
\tilde{k}_{I}^{j \rightarrow i}(\lambda)=\frac{C_{i j}^{2}}{2} G_{0, j}(\lambda)\left[1-G_{0, j}(\lambda) k_{I}^{j \rightarrow i}(\lambda)\right]^{-1} .
$$

Notice that $k_{I}$ does not depend on the real kernel, i.e., the imaginary kernels of the cavity influence functionals form a system of updates closed in themselves. This is also true without the assumptions that the process has been going on for a long time or under constant conditions.

The real part of the Vernon transform $\mathcal{W}$ is, however, a linear transformation of $k_{R}^{j \rightarrow i}$ to $\tilde{k}_{R}^{j \rightarrow i}$ which depends quadratically on $\tilde{k}_{I}^{j \rightarrow i}$. With the same assumptions as above it simplifies, on the Fourier domain, to

$$
\tilde{k}_{R}^{j \rightarrow i}(v)=A(v) k_{R}^{j \rightarrow i}(v)
$$

where $A(v)=\frac{4}{C_{i j}^{2}}\left|\tilde{k}_{I}^{j \rightarrow i}(v)\right|^{2}$. The real kernels of the cavity influence functions hence do not form a system closed in themselves. Furthermore, for a process over a finite time $\mathcal{W}$ also contains other terms which also depend on $\tilde{k}_{I}^{j \rightarrow i}$ and on initial conditions (bath temperature) but not on $k_{R}^{j \rightarrow i}$; see Appendix A. 


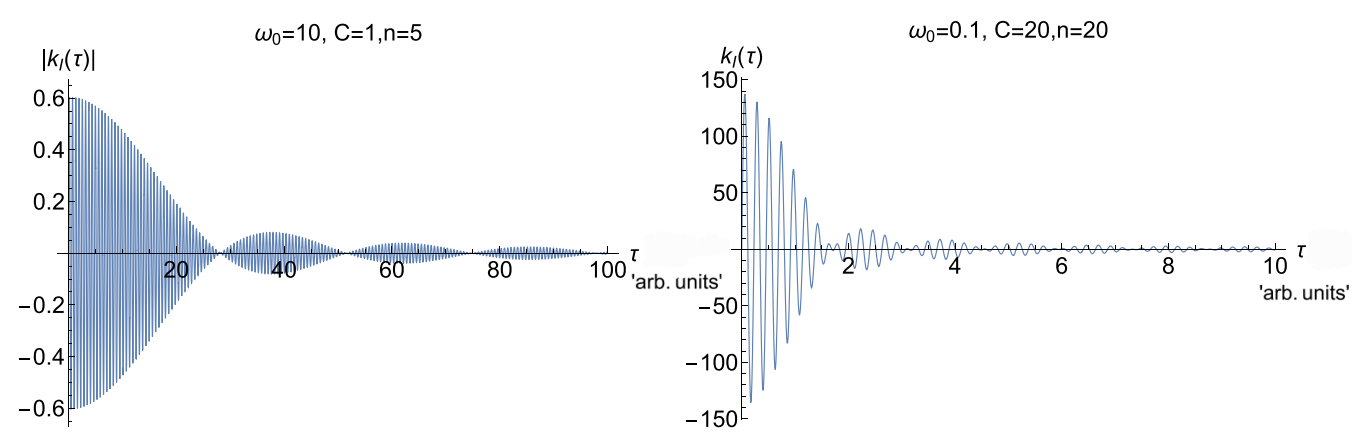

FIG. 2. Inverse Laplace transform of the fixed point kernel given by Eq. (C12). The parameters are: left panel- $\omega_{0}=10, C=1, n=5$, $m=\frac{1}{2}$; right panel- $\omega_{0}=0.1, C=20, n=20, m=\frac{1}{2}$.

In the specific case of random regular graphs where all oscillator frequencies and all interaction parameters are the same $\left(\omega_{j}=\omega\right.$ for all $j, C_{i j}=C$ for pairs $i$ and $j$ ) the equations written above admit a fixed point solution. Calling $n$ the number of neighbors of each node in this solution all kernels, everywhere in the network, are the same and follow

$$
k_{I}^{i+1}(\lambda)=\frac{(n-1) C^{2}}{2} G_{0}(\lambda)\left[1-G_{0}(\lambda) k_{I}^{i}(\lambda)\right]^{-1},
$$

where $i$ is now an iteration index. There is always a fixed point of this rational map for $C$ small enough. When it exists it is given by

$$
k_{I}^{*}(\lambda)=m \frac{\lambda^{2}+\omega^{2}}{4}\left[1-\sqrt{1-\frac{8(n-1) C^{2}}{m^{2}\left(\lambda^{2}+\omega^{2}\right)^{2}}}\right] .
$$

Since that $\omega^{2}$ has been defined as $\omega^{2}=\omega_{0}^{2}+\frac{n C}{m}$, the expression inside the square root in Eq. (24) is a decreasing function of $C$, positive for all $\lambda$ if either $n \geqslant 8$ or if $n<8$ and $C$ is less than a critical value $C^{*}(n)=\frac{m \omega_{0}^{2}}{\sqrt{8(n-1)}-n}$. For $n<8$ and $C>C^{*}(n)$ the fixed point still exists for $\lambda$ larger than $\lambda^{*}(C, n)=\omega_{0} \sqrt{\frac{C}{C^{*}}-1}$.

The first result on this quite simple example is hence that if $C<C^{*}$ every system in the uniform network behaves as if interacting with the same effective environment. We call this the ordered phase. The fixed point kernel $k_{I}^{*}$ in the time domain is illustrated in Fig. 2; a more detailed discussion can be found in the Appendices.

For $C>C^{*}(n)$ and $\lambda<\lambda^{*}(C, n)$ the BP messages [the functions $k_{I}^{i}(\lambda)$ ] do not converge as iteration index $i$ tends to infinity. In this setting it is therefore not consistent to assume that all incoming messages in Fig. 1 to be the same; there is nothing to synchronize them. As we will discuss in the following section, one can instead assume that each such $k_{I}^{k \rightarrow j}(\lambda)$ is a random number drawn from a probability $P\left[k_{I}^{k \rightarrow j}(\lambda)\right]$ and check if this distribution is preserved as $P\left[k_{I}^{j \rightarrow i}(\lambda)\right]$ (replica symmetric analysis of the BP update equations). In this regime, every system in the network behaves as if interacting with an environment, drawn randomly from the same ensemble of environments. We call this the dynamically disordered phase. The instances of this phase are complex, as there is no smooth function $f(t)$ which has a
Laplace transform $\tilde{f}(\lambda)$, for which when $\lambda<\lambda^{*}$ the values are independent random numbers.

This seemingly pathological property can be traced back to our neglecting the first time in the definition of $k_{I}(s, t-s)$. In the time domain $k_{I}^{i+1}(s, t-s)$ is related to $k_{I}^{i}\left(s-\tau_{I}, t-s\right)$ where $\tau_{I}$ is a characteristic time of the response function. When the process starts at time $t_{i}$ one can only iterate the quantum cavity in the form independent of the first time for $i^{\max } \approx \frac{s-t_{i}}{\tau_{I}}$ times until the initial conditions start to be felt. Therefore, the different components $k_{I}^{i}(s, \lambda)$ are actually correlated over a distance in $\lambda$ of size roughly $\Lambda^{-i^{\max }}$ where $\Lambda$ is a characteristic expansion rate of the Vernon transform. For the same reason, the potential divergence of the denominator on the right-hand side of Eq. (23), if in fact the incoming messages would be synchronized, is not a real difficulty. It can be traced back to the Fredholm integral equation defining $k_{I}^{i}(\lambda)$ (see Appendices), where each term in the series brings in an additional time delay. Over a finite time interval the individual terms are eventually modified, and the series converges.

However, when $k_{I}$ is at the fixed point Eq. (24) the multiplier $A(v)$, in front of the real kernel, depends on whether the expression in the square root in Eq. (24) (for $\lambda=i v$ ) is positive or negative. The second case pertains to a band of frequencies around $\omega$ with width linear in $C$ for small $C$. The value of $A(v)$ is 2 throughout the band, while it decreases down from 2 away from the band; see Fig. 3. In the ordered phase successive iteration of some Fourier components of $k_{R}$ hence increase without limit. By the same argument as above this pathological behavior can again be traced back to neglecting the first time; actually $k_{R}(s, v)$ only reaches size about $2^{\frac{s-t_{i}}{\tau_{I}}}$.

\section{DISORDER AND QUANTUM DYNAMICAL REPLICA SYMMETRY}

Understanding disorder in parameters is an important application of the cavity method. In this case, the fixed points of BP will then not be uniform, but the messages $m_{i \rightarrow j}$ and $n_{i \rightarrow j}$ will depend on the link in the network. While it is possible to solve for the coupled equations of all the messages in a whole network, a more compact description is in terms of self-consistency relations of probability distributions over messages. On the level of RS the real-time quantum cavity for 


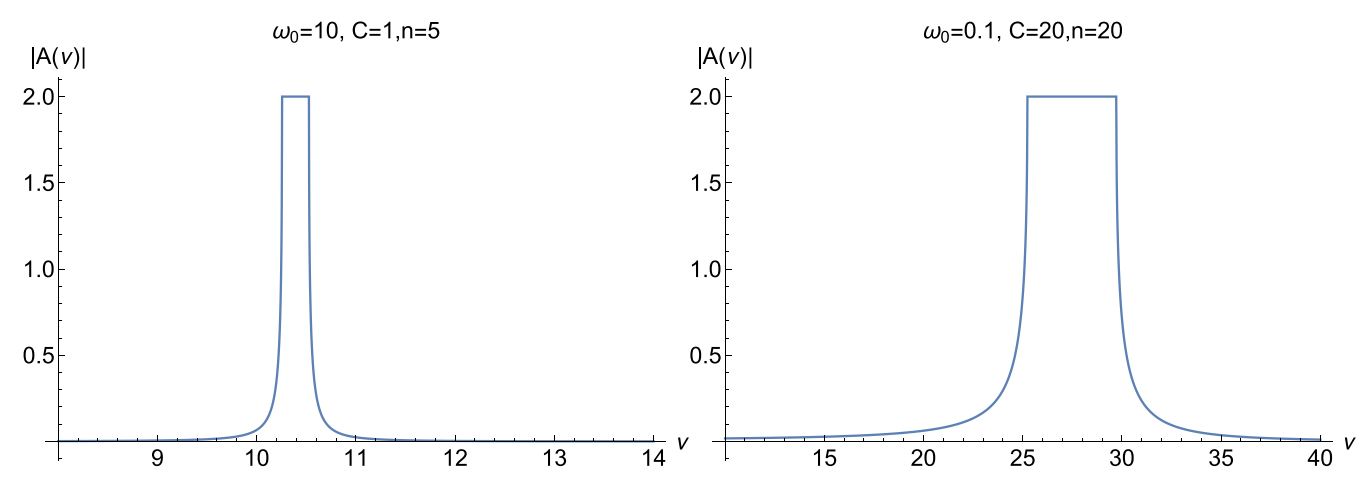

FIG. 3. The multiplier $A(v)$ in the real Vernon transformation $\mathcal{W}$, assuming the imaginary Vernon transform $\mathcal{V}$ has reached the fixed point given by Eq. (C12). Parameters are the same as in Fig. 2: left panel- $\omega_{0}=10, C=1, n=5, m=\frac{1}{2} ;$ right panel- $\omega_{0}=0.1, C=20, n=20$, $m=\frac{1}{2}$.

harmonic networks is hence described by probability distributions over the kernel pairs. As the BP update equation acts on $k_{I}$ kernel alone it is convenient to consider separately $P\left(\tilde{k}_{I}\right)$ and $Q\left(k_{I}\right)$, which satisfy RS cavity equations

$$
Q\left(k_{I}\right)=\sum_{k} \Lambda_{k} \int \prod_{k}\left[d \hat{k}_{I}^{k} P\left(\hat{k}_{I}^{k}\right)\right] \delta\left(k_{I}-\sum_{k} \hat{k}_{I}^{k}\right)
$$

and

$$
P\left(\hat{k}_{I}\right)=E_{D} \int d \hat{k}_{I} Q\left(k_{I}\right) \delta\left[\hat{k}_{I}-\mathcal{V}\left(k_{I}\right)\right],
$$

where $\Lambda_{k}$ is the probability of the neighborhood to be of size $k, E_{D}$ represents the average over the disorder, coupling or frequency distribution and $\mathcal{V}$ is the $n$ to $m$ step of the BP update determined by the Vernon transform. The kind of solution discussed in the previous sections in this formulation is described by $P$ 's and $Q$ 's that are $\delta$ functions. For a line or a random regular graph the size of every neighborhood is the same, and there are only identical terms in the sum in Eq. (25). It is easy to check that without disorder in the ordered phase the $\delta$ function distributions are stable, see Appendix D.

The most important effects of disorder can be discussed assuming that Eqs. (25) and (26) have solutions in terms of distributions of finite width with, roughly the size of disorder, (see Appendix E for a justification) and that the iteration of $\mathcal{W}$, the transformation acting on $k_{R}(v)$, amounts to multiplying by $\frac{4}{C^{2}}\left|k_{I}(v)\right|^{2}$. Therefore, the effect of many iterations on $k_{R}(v)$ is just a product of random numbers

$$
\begin{aligned}
\mathcal{W}^{k}\left(v, \omega_{k}, \ldots, \omega_{1}\right)= & \frac{4}{C^{2}}\left|k_{I}^{k}\left(v, \omega_{k}\right)\right|^{2} \frac{4}{C^{2}}\left|k_{I}^{k-1}\left(v, \omega_{k-1}\right)\right|^{2} \ldots \\
& \times \frac{4}{C^{2}}\left|k_{I}^{1}\left(v, \omega_{1}\right)\right|^{2}
\end{aligned}
$$

where the upper index above stands for the order of the iteration, and $\omega_{l}$ represents the disorder realized in iteration step $l$. The typical behavior of such products is well studied in random matrix theory; see, e.g., Ref. [43]. If all terms are similar and larger than one, then the product will typically also be larger than one and increase exponentially with the number of terms $k$. If, however, the terms are not similar and are mostly less than one, then the product will typically be small, and decrease exponentially in $k$.
In the problem at hand, the distinction between the two cases may be established in terms of the relation between the size of disorder in $\omega$ and the width of the plateau in the multiplier illustrated in Fig. 3. When the disorder is smaller than the plateau width, the behavior is similar to the case without disorder; for $v$ in the plateau the multiplier of $k_{R}(v)$ is around two, and those Fourier modes will grow leading to decoherence. In Appendix E 1 we verify analytically that such a solution to Eqs. (25) and (26) exists, and that the width of the distribution is proportional to the noise variance.

However, if the width of the disorder is larger than the plateau, then the multiplier will most often be small. In this phase $k_{R}(v)$ almost surely tends to zero upon iteration of the cavity equations. Every node in the network behaves as if interacting with an environment, drawn from an ensemble of environments, and every node has the property of finite dissipation (finite $k_{I}$ ) but no decoherence (zero $k_{R}$ ). Such an effective environment is qualitative different from a thermal bath at any temperature, where $k_{I}$ and $k_{R}$ would be linked by the fluctuation-dissipation theorem. In that case, for any nonzero $k_{I}$ (nonvanishing spectrum of the effective environment) there would be a nonzero $k_{R}$ which would lead to nonvanishing decoherence even when environment is in the ground state (zero bath temperature).

\section{CONCLUSIONS AND OUTLOOK}

In this work we have introduced a real-time version of the quantum cavity method. We have shown that when all systems in a network are harmonic oscillators interacting linearly, this real-time quantum cavity can be represented as transforms of Feynman-Vernon kernels. We have also shown that for uniform harmonic networks there is an ordered phase where one can solve explicitly for a fixed point of transformation. In this case, each single quantum harmonic oscillator behaves, for a long time dynamics, as if under the influence of a dissipation kernel of finite spectral support, and eventually arbitrarily strong decoherence, such a network will eventually behave entirely classically. We have also presented how this technique can be extended to study an ensemble average over a disordered system under the replica symmetry ansatz. We know that disorder in equilibrium models induce an Anderson transition on locally treelike graphs $[5,7,44]$. For 
small disorder states are delocalized and influence propagate though the network, while for large disorder states are localized. We have shown that a similar transition also takes place for our real-time problem. With disorder each system perceives the network as an instance of an ensemble of effective environments. As in the uniform case, for small disorder the system presents finite decoherence through the whole network - delocalized state-while, when the disorder is large enough, these effective environments have the unexpected property of finite dissipation (finite imaginary FeynmanVernon kernel $k_{I}$ ) but almost surely zero decoherence (zero real Feynman-Vernon kernel $k_{R}$ ) retaining quantum coherence locally-localized state. We therefore believe that this transition may be a reflection, in the real-time dynamics of a disordered network, of the equilibrium Anderson transition.

Our results constitute a first step toward a more detailed and deeper characterization of the dynamics of open quantum networks. For example, while in this work we focused on the regime of stationary parameters, in most quantum computational applications, the network parameters would change in time. This would entail full two-time kernels $k_{I}$ and $k_{R}$ to describe the effective environment. Also, the examples we have considered are networks of harmonic oscillators, as they yield explicit solutions of the real-time quantum cavity in closed form. While the challenges in extending these investigations to qubits or other systems are significant, the Vernon transform is defined also for environments that are not harmonic oscillators [40] these can be treated as done in this work. While keeping an infinite tower of higher-order Feynman-Vernon kernels may be impractical, one can consider truncations, the analogues of which have found many applications in classical information processing $[45,46]$.

\section{ACKNOWLEDGMENTS}

E.A. thanks Foundation for Polish Science through TEAMNET project (Contract No. POIR.04.04.00-00-17C1/18-00) for support during the initial phase of this work and Swedish Research Council Grant No. 2020-04980 during its completion phase. J.T. was supported by the European Research Council Grant No. 742104.

\section{APPENDIX A: THE VERNON TRANSFORM IN THE TIME DOMAIN AND ON THE LAPLACE TRANSFORM SIDE}

This Appendix contains further details on the calculation outlined in the main body of the paper. It starts with a presentation of the Vernon transform ([41], Appendix V); a presentation of this material by two of us can also be found in Ref. [47]. As noted in main paper the Vernon transform has been rediscovered at least once in the later literature [42].

In contrast to the presentation in the main paper we now keep the time dependence throughout, to eventually write the Vernon transform for a time-stationary situation on the Laplace side. The point of departure is then Eq. (14), the path integral expressing the Vernon transform. For illustration, for the moment we do not require the initial state to be symmetric. We introduce for simplicity new variables

$$
\begin{aligned}
& \bar{X}_{j}(t)=X_{j}(t)+Y_{j}(t), \\
& \Delta_{j}(t)=X_{j}(t)-Y_{j}(t),
\end{aligned}
$$

and similarly for the target node $i$ and the initial and final state on $j$. In this way one can write

$$
\begin{aligned}
e^{\frac{i}{\hbar} \tilde{F}_{j \rightarrow i}\left[\bar{X}_{i}, \Delta_{i}\right]}= & \int \delta\left(\Delta_{j}^{T}\right) \exp \left\{\frac{i}{\hbar} \int_{\tau}^{T}\left[\left(\frac{m}{2} \dot{\bar{X}}_{j} \dot{\Delta}_{j}-\frac{m \omega^{2}}{2} \bar{X}_{j} \Delta_{j}+\frac{C_{i j}}{2} \bar{X}_{i} \Delta_{j}+\frac{C_{i j}}{2} \Delta_{i} \bar{X}_{j}\right)\right] d t\right\} \\
& \times \exp \left\{\frac{i}{\hbar} \int_{\tau}^{T} \int_{\tau}^{t} k_{I}^{j \rightarrow i}(s, t-s) \Delta_{j}(t) \bar{X}_{j}(s) d t d s-\frac{1}{\hbar} \int_{\tau}^{T} \int_{\tau}^{t} k_{R}^{j \rightarrow i}(t, s) \Delta_{j}(t) \Delta_{j}(s) d t d s\right\} \\
& \times \frac{1}{N} \exp \left[-\left(A^{\prime}\left(\bar{X}_{j}^{\tau}\right)^{2}+2 B^{\prime} \bar{X}_{j}^{\tau} \Delta_{j}^{\tau}+C^{\prime}\left(\Delta_{j}^{\tau}\right)^{2}\right) / 2\right] \mathcal{D} \bar{X}_{j} \mathcal{D} \Delta_{j} d \Delta_{j}^{\tau} d \Delta_{j}^{T} d \bar{X}_{j}^{\tau} d \bar{X}_{j}^{T},
\end{aligned}
$$

where $A^{\prime}=\frac{1}{4}(A+2 B+C), C^{\prime}=\frac{1}{4}(A-2 B+C)$, and $B^{\prime}=\frac{1}{2}(A-C)$. Requiring that the initial state does not mix $\bar{x}_{j}$ and $\Delta_{j}$ leads to $B^{\prime}=0$ and $A^{\prime}=\frac{1}{2}(A+B)$ and $C^{\prime}=\frac{1}{2}(A-B)$. Note that the normalization $\frac{1}{N} \int e^{-\frac{1}{2}\left(A^{\prime} \bar{x}^{2}\right)} d \bar{x}=2$. A thermal state at inverse temperature $\beta$ has $A=C=\frac{m_{j} \omega_{j}}{\hbar} \operatorname{coth} \beta \hbar \omega_{j}$ and $B=-\frac{m_{j} \omega_{j}}{\hbar} \sinh ^{-1} \beta \hbar \omega_{j}$, and hence $A^{\prime}=\frac{m_{j} \omega_{j}}{2 \hbar} \tanh \frac{\beta \hbar \omega_{j}}{2}$ and $C^{\prime}=\frac{m_{j} \omega_{j}}{2 \hbar} \operatorname{coth} \frac{\beta \hbar \omega_{j}}{2}$. The assumption of an initially symmetric Gaussian state is hence equivalent to assuming an initial thermal state where the two parameters $A^{\prime}$ and $C^{\prime}$ set a length scale $\ell=\frac{2 \hbar}{m_{j} \omega_{j}}=\frac{1}{\sqrt{A^{\prime} C^{\prime}}}$ and an inverse temperature $\beta=\frac{2}{\hbar \omega_{j}}$ tanh ${ }^{-1} \sqrt{\frac{A^{\prime}}{C^{\prime}}}$. We will drop the primes on $A^{\prime}$ and $C^{\prime}$ from now on.

The first step is now to integrate the term $\dot{\bar{X}}_{j} \dot{\Delta}_{j}$ by parts which gives

$$
\int_{\tau}^{T} \dot{\bar{X}}_{j} \dot{\Delta}_{j} d t=\dot{\Delta}_{j}^{T} \bar{X}_{j}^{T}-\dot{\Delta}_{j}^{\tau} \bar{X}_{j}^{\tau}-\int_{\tau}^{T} \bar{X}_{j} \ddot{\Delta}_{j} d t
$$

from which follows

$$
e^{\frac{i}{\hbar} \tilde{F}_{j \rightarrow i}\left[\bar{X}_{i}, \Delta_{i}\right]}=\frac{1}{2} \int \delta\left(\Delta_{j}^{T}\right) \exp \left\{\frac{i}{\hbar} \int_{\tau}^{T}\left[\left(-\frac{m}{2} \bar{X}_{j} \ddot{\Delta}_{j}-\frac{m \omega^{2}}{2} \bar{X}_{j} \Delta_{j}+\frac{C_{i j}}{2} \bar{X}_{i} \Delta_{j}+\frac{C_{i j}}{2} \Delta_{i} \bar{X}_{j}\right)\right] d t\right\}
$$




$$
\begin{aligned}
& \times \exp \left\{\frac{i}{\hbar} \int_{\tau}^{T} \int_{\tau}^{t} k_{I}^{j \rightarrow i}(s, t-s) \Delta_{j}(t) \bar{X}_{j}(s) d t d s-\frac{1}{\hbar} \int_{\tau}^{T} \int_{\tau}^{t} k_{R}^{j \rightarrow i}(t, s) \Delta_{j}(t) \Delta_{j}(s) d t d s\right\} \\
& \times \frac{1}{N} \exp \left\{-\left[A \bar{X}_{j}^{\tau^{2}}+C\left(\Delta_{j}^{\tau}\right)^{2}\right] / 2\right\} \exp \frac{i m}{2 \hbar}\left(\dot{\Delta}_{j}^{T} \bar{X}_{j}^{T}-\dot{\Delta}_{j}^{\tau} \bar{X}_{j}^{\tau}\right) \mathcal{D} \bar{X}_{j} \mathcal{D} \Delta_{j} d \Delta_{j}^{\tau} d \Delta_{j}^{T} d \bar{X}_{j}^{\tau} d \bar{X}_{j}^{T} .
\end{aligned}
$$

The prefactor $\frac{1}{2}$ is the Jacobian of the change of variables at the initial time; the other (functional) Jacobian is included in the path integral measure. The initial state is Gaussian, and we can integrate over $\bar{X}_{j}$. This gives $\frac{1}{N} \int d \bar{X}_{j}^{\tau} e^{-\frac{1}{2} A^{\prime} \bar{X}_{j}^{\tau}{ }^{2}-\frac{i m}{2 \hbar} \dot{\Delta}_{j}^{\tau} \bar{X}_{j}^{\tau}}=$ $2 e^{-\frac{m^{2}}{8 \hbar^{2} \AA^{\prime}}\left(\Delta_{j}^{\tau}\right)^{2}}$ where the factor 2 cancels in above. The corresponding integral over the final state fixes the final velocity for $\Delta_{j}$, that is $\int d \bar{X}_{j}^{T} \exp ^{\frac{i m}{2 \hbar}} \dot{\Delta}_{j}^{T} \bar{X}_{j}^{T}=\delta\left(\dot{\Delta}_{j}^{T}\right)$. The remaining integrals over $\bar{X}_{j}$ at intermediate times give a $\delta$ functional

$$
\delta\left[g\left(\Delta_{j}\right)(t)\right]
$$

where

$$
g\left(\Delta_{j}\right)(t)=\frac{m}{2} \ddot{\Delta}_{j}(t)+\frac{m \omega^{2}}{2} \Delta_{j}(t)-\frac{C_{i j}}{2} \Delta_{i}(t)-\int_{t}^{T} k_{I}^{j \rightarrow i}(t, s-t) \Delta_{j}(s) d s .
$$

The integral over the deviation path $\Delta_{j}(t)$ hence has support on a classical path which satisfies final conditions $\Delta_{j}=\dot{\Delta}_{j}=0$, and equations of motion $g\left(\Delta X_{j}\right)(t)=0$. It is convenient to call this auxiliary path $Q(t)$. The double path integral in Eq. (A5) hence gives

$$
\frac{i}{\hbar} \tilde{F}_{j \rightarrow i}\left[\bar{X}_{i}, \Delta X_{i}\right]=\frac{i}{\hbar} \int_{\tau}^{T} \frac{C_{i j}(t)}{2} \bar{X}_{i}(t) Q(t) d t-\frac{1}{2 \hbar} \int_{\tau}^{T} \int_{\tau}^{T} k_{R}^{j \rightarrow i}(t, s) Q(t) Q(s)-\frac{1}{2} C q^{2}-\frac{m^{2}}{8 \hbar^{2} A}(\dot{q})^{2},
$$

where by $q$ we mean the initial position of the auxiliary classical path, i.e., $Q(\tau) . Q(t)$ depends on the deviation path $\Delta X_{i}(s)$ for all values of $s$ larger than $t$. This is because $Q$ satisfies final conditions as $s=T$ while its initial conditions at $s=\tau$ are not given. It is further clear that $Q(t)$ is a linear functional $\Delta X_{i}(s)$ for $s \in[t, T]$. This linear functional can be represented by a kernel

where

$$
Q(t)=\int_{t}^{T} G(t, s-t) C_{i j}(s) \Delta X_{j}(s) d s,
$$

$$
G(t, s-t)=G_{0}(s-t)+\int_{t}^{T} d t_{1} \int_{t_{1}}^{\infty} d t_{2} G_{0}\left(t_{1}-t\right) k_{I}^{k \rightarrow j}\left(t_{1}, t_{2}-t_{1}\right) G\left(t_{2}, s-t_{2}\right) .
$$

As shown in Appendix B there is at least as a formal power series a kernel which satisfies $G(t, s-t)=0$ for $s<t$. Substituting Eq. (A9) in Eq. (A8) we have the kernels of the transformed Feynman-Vernon action as

$$
\begin{gathered}
\tilde{k}_{I}^{j \rightarrow i}(t, s-t)=\frac{1}{2} C_{i j}(t) C_{i j}(s) G(t, s-t), \\
\tilde{k}_{R}^{j \rightarrow i}(t, s)=C_{i j}(t) C_{i j}(s) \int_{\tau}^{t} \int_{\tau}^{s} k_{R}^{j \rightarrow i}\left(t^{\prime}, s^{\prime}\right) G\left(t^{\prime}, t-t^{\prime}\right) G\left(s^{\prime}, s-s^{\prime}\right) d s^{\prime} d t^{\prime}+C_{i j}(t) C_{i j}(s) \hbar C G(\tau, t+\tau) G(\tau, s+\tau) \\
+\left.\left.C_{i j}(t) C_{i j}(s) \frac{1}{\hbar A} \frac{d G(r, t-r)}{d r}\right|_{r=\tau} \frac{d G(r, s-r)}{d r}\right|_{r=\tau} .
\end{gathered}
$$

The last two terms are zero if the auxiliary path $Q(t)$ returns to rest at the origin at $t \rightarrow \tau$. This should be so whenever the kernel $k_{I}^{j \rightarrow i}(t, s-t)$ behaves as friction and when the process goes on for infinite time $(\tau=-\infty)$, and when the drive $\left[C_{i j}(t)\right]$ vanishes before some turn-on time $t_{i}$. In any case, if the response function $G(\tau, t+\tau)$ has essentially finite support in $t$, then these two terms will only give a boundary contribution and will not matter when $t$ and $s$ are sufficiently larger than $\tau$.

In this way we have formally established the BP update equation as an integral transformation on Feynman-Vernon kernels given by Eq. (A11) and the first line of Eq. (A12). Equations (A11) and (A12) can be closed through the use of Eq. (10). Concretely we write them as

$$
\begin{aligned}
k_{I}^{j \rightarrow i}(t, s-t)= & \sum_{k \in \partial j / i} \tilde{k}_{I}^{k \rightarrow j}(t, s-t)=\sum_{k \in \partial j / i} \frac{1}{2} C_{k j}(t) C_{k j}(s) G(t, s-t), \\
k_{R}^{j \rightarrow i}(t, s)= & \sum_{k \in \partial j / i} \tilde{k}_{R}^{k \rightarrow j}(t, s)=\sum_{k \in \partial j / i} C_{k j}(t) C_{k j}(s) \\
& \times \int_{-\infty}^{t} \int_{-\infty}^{s} k_{R}^{k \rightarrow j}\left(t^{\prime}, s^{\prime}\right) G\left(t^{\prime}, t-t^{\prime}\right) G\left(s^{\prime}, s-s^{\prime}\right) d s^{\prime} d t^{\prime} .
\end{aligned}
$$

Note that the information about the graph structure is given through the introduction of the $\sum_{k \in \partial j / i}$. 


\section{APPENDIX B: REPRESENTATION OF THE KERNEL $G$}

Our goal of this Appendix is to derive an explicit representation of the kernel $G$ defined in Eq. (A9) in the preceding Appendix. This kernel is to relate a function $Q(t)$ to a source term $C_{i j}(s) \Delta X_{i}(s)$ for all $s>t$. Values of the source at times $s$ earlier than $t$ have no influence on $Q(t)$. The solution must therefore satisfy $G(t, s-t)=0$ when $s<t$.

$Q(t)$ is determined by the equation of motion of an externally driven harmonic oscillator with non-Markovian damping [given in the preceding Appendix as Eq. (A7) and below as Eq. (B1)] and final conditions $Q(T)=\dot{Q}(T)=0$. For an infinite time interval $(\tau=-\infty$ and $T=\infty)$ it is convenient to assume that $C_{i j}(s)$ vanishes for $s>t_{f}$ as well as for $s<t_{i}$. The first means that $Q(t)$ must also vanish for $t>t_{f}$. The second means that for those values $Q$ satisfies an autonomous integro-differential equation without drive. That is, if we know $Q$ in the interval $\left[t_{i}: t_{f}\right]$, then $Q(t)$ for $t<t_{i}$ follows as a consequence.

For convenience we restate the equation satisfied by $Q$ representing oscillator $k$ driven by oscillator $j$ :

$$
\begin{aligned}
& \frac{m}{2} \ddot{Q}+\frac{m \omega^{2}}{2} Q-\frac{1}{2} C_{k j}(t) \Delta_{j}(t) \\
& \quad-\int_{t}^{\infty} d s k_{I}^{k \rightarrow j}(t, s-t) Q(s)=0 .
\end{aligned}
$$

To emphasize that $k_{I}^{k \rightarrow j}(t, s-t)$ vanishes when the second argument is negative we write out explicitly a Heaviside function $\Theta(s-t)$. We start introducing the Fourier transform of

$$
Q(t)=\frac{1}{2 \pi} \int_{-\infty}^{\infty} d v e^{-i v t} \hat{Q}(v)
$$

and

$$
k_{I}^{k \rightarrow j}(t, s-t) \Theta[s-t]=\frac{1}{2 \pi} \int_{-\infty}^{\infty} d \mu e^{-i \mu(s-t)} \hat{k}_{I^{-}}^{k \rightarrow j}(t, \mu),
$$

where $\mu$ should have an infinitesimal positive imaginary part. Substituted in Eq. (B1) this leads to

$$
\begin{aligned}
& \frac{1}{2 \pi} \int d v e^{-i v t}\left[\frac{m}{2}\left(-v^{2}+\omega^{2}\right) \hat{\Delta}_{k}(v)\right. \\
& \left.\quad-\frac{1}{2}\left[C_{k j} \Delta_{j}\right]_{v}-\hat{k}_{I^{-}}^{k \rightarrow j}(t,-v) \hat{\Delta}_{k}(v)\right]=0,
\end{aligned}
$$

where $v$ should have an infinitesimal negative imaginary part. Integrating over time as $\int d t e^{i \kappa t}(\cdots)$

$$
\begin{gathered}
\frac{1}{2 \pi} \int d t e^{i \kappa t} \int d v e^{-i v t}\left[\frac{m}{2}\left(-v^{2}+\omega^{2}\right) \hat{\Delta}_{k}(v)\right. \\
\left.-\frac{1}{2}\left[C_{k j} \Delta_{j}\right]_{v}-\hat{k}_{I^{-}}^{k \rightarrow j}(t,-v) \hat{\Delta}_{k}(v)\right]=0,
\end{gathered}
$$

and defining

$$
\hat{k}_{I^{-}}^{k \rightarrow j}(\kappa-v,-v)=\int_{-\infty}^{\infty} d t e^{i(\kappa-v) t} \hat{k}_{I^{-}}^{k \rightarrow j}(t,-v)
$$

one finds

$$
\begin{aligned}
& {\left[\frac{m}{2}\left(-\kappa^{2}+\omega^{2}\right) \hat{Q}(\kappa)-\frac{1}{2}\left[C_{k j} \Delta_{j}\right]_{\kappa}\right.} \\
& \left.\quad-\frac{1}{2 \pi} \int_{-\infty}^{\infty} d \nu \hat{\hat{k}}_{I^{-}}^{k \rightarrow j}(\kappa-v,-v) \hat{Q}(v)\right]=0,
\end{aligned}
$$

where $\kappa$ should have an infinitesimal negative imaginary part.

We can rewrite the preceding expression as

$$
\hat{Q}(\kappa)=\frac{\frac{1}{2}\left[C_{k j} \Delta_{j}\right]_{\kappa}}{\frac{m}{2}\left(\omega^{2}-\kappa^{2}\right)}+\frac{\frac{1}{2 \pi} \int_{-\infty}^{\infty} d \nu \hat{\hat{k}}_{I^{-}}^{k \rightarrow j}(\kappa-v,-v) \hat{Q}(v)}{\frac{m}{2}\left(\omega^{2}-\kappa^{2}\right)},
$$

which is a Fredholm singular integral. It can be written

$$
\hat{Q}(\kappa)=f(\kappa)+\lambda \int_{-\infty}^{\infty} d \nu K(\kappa, v) \hat{Q}(\nu) .
$$

Applying the method of successive iterated approximations one finds

$$
\begin{aligned}
\hat{Q}(\kappa)= & f(\kappa)+\lambda \int_{-\infty}^{\infty} d \nu K(\kappa, v) f(\nu)+\lambda^{2} \int_{-\infty}^{\infty} \int_{-\infty}^{\infty} d \nu d v_{1} K(\kappa, v) K\left(\nu, v_{1}\right) f\left(v_{1}\right) \\
& +\lambda^{2} \int_{-\infty}^{\infty} \int_{-\infty}^{\infty} \int_{-\infty}^{\infty} d \nu d v_{1} d v_{2} K(\kappa, v) K\left(\nu, v_{1}\right) K\left(v_{1}, v_{2}\right) f\left(v_{2}\right)+\cdots
\end{aligned}
$$

Substituting for our $f(\kappa)$ and $K(\kappa, v)$ one finds

$$
\begin{aligned}
& \hat{Q}(\kappa)=\frac{\frac{1}{2}\left[C_{k j} \Delta_{j}\right]_{\kappa}}{\frac{m}{2}\left(\omega^{2}-\kappa^{2}\right)}+\int_{-\infty}^{\infty} d \nu \frac{\frac{1}{2 \pi} \hat{k}_{I^{-}}^{k \rightarrow j}(\kappa-v,-v)}{\frac{m}{2}\left(\omega^{2}-\kappa^{2}\right)} \frac{\frac{1}{2}\left[C_{k j} \Delta_{j}\right]_{\nu}}{\frac{m}{2}\left(\omega^{2}-\nu^{2}\right)} \\
& +\int_{-\infty}^{\infty} \int_{-\infty}^{\infty} d v d v_{1} \frac{\frac{1}{2 \pi} \hat{\hat{k}}_{I^{-}}^{k \rightarrow j}(\kappa-v,-v)}{\frac{m}{2}\left(\omega^{2}-\kappa^{2}\right)} \frac{\frac{1}{2 \pi} \hat{k}_{I^{-}}^{k \rightarrow j}\left(v-v_{1},-v_{1}\right)}{\frac{m}{2}\left(\omega^{2}-v^{2}\right)} \frac{\frac{1}{2}\left[C_{k j} \Delta_{j}\right]_{\nu_{1}}}{\frac{m}{2}\left(\omega^{2}-v_{1}^{2}\right)} \\
& +\int_{-\infty}^{\infty} \int_{-\infty}^{\infty} \int_{-\infty}^{\infty} d v d v_{1} d v_{2} \frac{\frac{1}{2 \pi} \hat{k}_{I^{-}}^{k \rightarrow j}(\kappa-v,-v)}{\frac{m}{2}\left(\omega^{2}-\kappa^{2}\right)} \frac{\frac{1}{2 \pi} \hat{k}_{I^{-}}^{k \rightarrow j}\left(v-v_{1},-v_{1}\right)}{\frac{m}{2}\left(\omega^{2}-v^{2}\right)} \frac{\frac{1}{2 \pi} \hat{k}_{I^{-}}^{k \rightarrow j}\left(v_{1}-v_{2},-v_{2}\right)}{\frac{m}{2}\left(\omega^{2}-v_{1}^{2}\right)} \frac{\frac{1}{2}\left[C_{k j} \Delta_{j}\right]_{\nu_{2}}}{\frac{m}{2}\left(\omega^{2}-v_{2}^{2}\right)} \\
& +\cdots \text {. }
\end{aligned}
$$


The above expression can be written as $Q(t)=\int_{-\infty}^{\infty} d s \frac{1}{2} G(t, s) C_{k j}(s) \Delta_{j}(s)$, where $G(t, s)$ stands for the iterated sum

$$
\begin{aligned}
G(t, s) & =\left[\frac{1}{2 \pi} \int_{-\infty}^{\infty} d \kappa e^{-i \kappa t} \frac{1}{\frac{m}{2}\left(\omega^{2}-\kappa^{2}\right)} e^{i \kappa s}+\frac{1}{2 \pi} \int_{-\infty}^{\infty} d \kappa e^{-i \kappa t} \frac{1}{\frac{m}{2}\left(\omega^{2}-\kappa^{2}\right)} \int_{-\infty}^{\infty} d v \frac{\frac{1}{2 \pi} \hat{k}_{I^{-}}^{k \rightarrow j}(\kappa-\nu,-v)}{\frac{m}{2}\left(\omega^{2}-v^{2}\right)} e^{i \nu s}\right. \\
& \left.+\frac{1}{2 \pi} \int_{-\infty}^{\infty} d \kappa e^{-i \kappa t} \frac{1}{\frac{m}{2}\left(\omega^{2}-\kappa^{2}\right)} \int_{-\infty}^{\infty} \int_{-\infty}^{\infty} d \nu d v_{1} \frac{\frac{1}{2 \pi} \hat{k}_{I^{-}}^{k \rightarrow j}(\kappa-v,-v)}{\frac{m}{2}\left(\omega^{2}-v^{2}\right)} \frac{\frac{1}{2 \pi} \hat{k}_{I^{-}}^{k \rightarrow j}\left(\nu-v_{1},-v_{1}\right)}{\frac{m}{2}\left(\omega^{2}-v_{1}^{2}\right)} e^{i \nu_{1} s}+\cdots\right]
\end{aligned}
$$

The first (zero-order) term in the sum

$$
G_{0}(t, s)=\frac{1}{2 \pi} \int_{-\infty}^{\infty} d \kappa e^{i \kappa(s-t)} \frac{1}{\frac{m}{2}\left(\omega^{2}-\kappa^{2}\right)} .
$$

When $\kappa$ has infinitesimal negative imaginary part and when $s-t$ is negative, the integral can be closed in the lower half plane, and is zero. When $s-t$ is positive the integral can be closed in the upper complex plane and is $\frac{2}{m \omega} \sin \omega(s-t)$.

The next (first-order) term is

$$
\begin{aligned}
G_{1}(t, s) & =\frac{1}{2 \pi} \int_{-\infty}^{\infty} d \kappa e^{-i \kappa t} \frac{1}{\frac{m}{2}\left(\omega^{2}-\kappa^{2}\right)} \int_{-\infty}^{\infty} d v \frac{e^{i v s}}{\frac{m}{2}\left(\omega^{2}-v^{2}\right)} \frac{1}{2 \pi} \hat{k}_{I^{-}}^{k \rightarrow j}(\kappa-v,-v) \\
& =\frac{1}{2 \pi} \int_{-\infty}^{\infty} d \kappa \frac{e^{-i \kappa t}}{\frac{m}{2}\left(\omega^{2}-\kappa^{2}\right)} \int_{-\infty}^{\infty} d \nu \frac{e^{i v s}}{\frac{m}{2}\left(\omega^{2}-v^{2}\right)} \frac{1}{2 \pi} \int_{-\infty}^{\infty} d t_{1} e^{i(\kappa-v) t_{1}} \hat{k}_{I^{-}}^{k \rightarrow j}\left(t_{1},-v\right) \\
& =\frac{1}{2 \pi} \int_{-\infty}^{\infty} d \kappa \frac{e^{-i \kappa t}}{\frac{m}{2}\left(\omega^{2}-\kappa^{2}\right)} \int_{-\infty}^{\infty} d \nu \frac{e^{i v s}}{\frac{m}{2}\left(\omega^{2}-v^{2}\right)} \frac{1}{2 \pi} \int_{-\infty}^{\infty} d t_{1} e^{i(\kappa-v) t_{1}} \int_{-\infty}^{\infty} d t_{2} e^{-i v\left(t_{2}-t_{1}\right)} k_{I}^{k \rightarrow j}\left(t_{1}, t_{2}-t_{1}\right) \Theta\left(t_{2}-t_{1}\right)
\end{aligned}
$$

where the definitions of $\hat{\hat{k}}_{I^{-}}^{k \rightarrow j}(\kappa-v,-v)$ and $\hat{k}_{I}^{k \rightarrow j}(t,-v)$ given in Eqs. (B6) and (B6) were used. In the above some term can be rearranged to give

$$
\begin{aligned}
G_{1}(t, s) & =\frac{1}{2 \pi} \int_{-\infty}^{\infty} d t_{1} \int_{-\infty}^{\infty} d t_{2} \frac{1}{2 \pi} k_{I}^{k \rightarrow j}\left(t_{1}, t_{2}-t_{1}\right) \Theta\left(t_{2}-t_{1}\right) \int_{-\infty}^{\infty} d \kappa \frac{e^{-i \kappa\left(t-t_{1}\right)}}{\frac{m}{2}\left(\omega^{2}-\kappa^{2}\right)} \int_{-\infty}^{\infty} d v \frac{e^{i v\left(s-t_{2}\right)}}{\frac{m}{2}\left(\omega^{2}-v^{2}\right)} \\
& =\int_{-\infty}^{\infty} d t_{1} \int_{t_{1}}^{\infty} d t_{2} k_{I}^{k \rightarrow j}\left(t_{1}, t_{2}-t_{1}\right) \frac{1}{2 \pi} \int_{-\infty}^{\infty} d \kappa \frac{e^{-i \kappa\left(t-t_{1}\right)}}{\frac{m}{2}\left(\omega^{2}-\kappa^{2}\right)} \frac{1}{2 \pi} \int_{-\infty}^{\infty} d v \frac{e^{i v\left(s-t_{2}\right)}}{\frac{m}{2}\left(\omega^{2}-v^{2}\right)} \\
& =\int_{-\infty}^{\infty} d t_{1} \int_{t_{1}}^{\infty} d t_{2} k_{I}^{k \rightarrow j}\left(t_{1}, t_{2}-t_{1}\right) G_{0}\left(t_{1}-t\right) G_{0}\left(s-t_{2}\right)
\end{aligned}
$$

Successive repetition of the above procedure leads to the following expression:

$$
\begin{aligned}
G(t, s-t)= & G_{0}(s-t)+\int_{t}^{T} d t_{1} \int_{t_{1}}^{T} d t_{2} G_{0}\left(t_{1}-t\right) k_{I}^{k \rightarrow j} \\
& \times\left(t_{1}, t_{2}-t_{1}\right) G\left(t_{2}, s-t_{2}\right),
\end{aligned}
$$

which is Eq (18). In general, the above one-sided functional equation does not have a convenient closed-form solution. However, if one assumes that both $k_{I}^{k \rightarrow j}(t, s-t)$ and $G(t, s-$ $t$ ) depend only on their second argument and essentially vanish when it is large enough, then one has the considerably simpler relation

$$
\begin{aligned}
G(s-t)= & G_{0}(s-t)+\int_{t}^{\infty} d t_{1} \int_{t_{1}}^{\infty} d t_{2} G_{0} \\
& \times\left(t_{1}-t\right) k_{I}^{k \rightarrow j}\left(t_{2}-t_{1}\right) G\left(s-t_{2}\right)
\end{aligned}
$$

valid when $s$ (the largest time in above) is considerably smaller than $T$ (the final time). Since this equation involves a convolution, it can be conveniently written in the Laplace domain as

$$
\tilde{G}(\lambda)=\tilde{G}_{0}(\lambda)+\tilde{G}_{0}(\lambda) k_{I}^{k \rightarrow j}(\lambda) \tilde{G}(\lambda),
$$

where for simplicity we used the same symbol for $k_{I}^{k \rightarrow j}$ in the time domain and in the Laplace domain. The Laplace transform $\tilde{G}_{0}(\lambda)$ is given by

$$
\tilde{G}_{0}(\lambda)=\int_{0}^{\infty} e^{-\lambda t} \frac{2}{m \omega} \sin \omega t d t=\frac{2}{m} \frac{1}{\lambda^{2}+\omega^{2}} .
$$

This is twice the response function of the harmonic oscillator as conventionally defined.

\section{APPENDIX C: FIXED POINT FOR CONSTANT INTERACTIONS ON $\boldsymbol{n}$-REGULAR RANDOM GRAPHS}

The goal of this Appendix is to make an explicit computation of Eq. (A13) in a specific model. We will present two approaches, one relying on the known representation of the Laplace transform function, and one of a sine transform analogous to the the Mehler-Sonine representation of the Bessel function. We consider a set of oscillators interacting with constant couplings, what one may call a ferromagnetic case. In the 
present context constant interaction means that all functions $C_{i j}(t)$ are the same and do not depend on time. Moreover, we will assume that oscillators are placed in an $n$-regular random graphs, i.e., a graph where all vertices have the same number (n) of neighbors. In such a setting there can be a deterministic replica symmetric phase of the cavity equations where all cavity messages are the same. In this Appendix we will consider the corresponding fixed point and the corresponding message.

In this case, it is easy to show that

$$
\begin{gathered}
\tilde{k}_{I}^{k \rightarrow j}(t, s-t)=\frac{1}{2} C_{k j} C_{k j} G^{k \rightarrow j}(t, s-t), \\
\tilde{k}_{R}^{k \rightarrow j}(t, s)=C_{k j} C_{k j} \int_{-\infty}^{t} \int_{-\infty}^{s} k_{R}^{k \rightarrow j}\left(t^{\prime}, s^{\prime}\right) G^{k \rightarrow j} \\
\times\left(t^{\prime}, t-t^{\prime}\right) G^{k \rightarrow j}\left(s^{\prime}, s-s^{\prime}\right) .
\end{gathered}
$$

These expression apparently suggest that the sign of the couplings $C_{i j}$ is irrelevant, they always appear squared. However, we have to remember that in above we have implicitly used the relation $\omega^{2}=\omega_{0}^{2}+\frac{n C}{m}$ which enters in the bare response function $G_{0}$, which in turn enters in $G$. $C$ can be taken arbitrarily large positive, but not smaller than $-\frac{m \omega_{0}^{2}}{n}$, as otherwise the total potential is not positive definite and the system has no ground state. We now turn to the two different ways in which the fixed point of the imaginary kernel can be derived.

\section{First version of the calculation}

We start by summing the twinning relation Eq. (B18) which gives

$$
\begin{aligned}
\tilde{G}(\lambda) & =\tilde{G}_{0}(\lambda)+\tilde{G}_{0}(\lambda) k_{I}^{k \rightarrow j}(\lambda) \tilde{G}(\lambda) \\
& =\tilde{G}_{0}(\lambda)\left[1-\tilde{G}_{0}(\lambda) k_{I}^{k \rightarrow j}(\lambda)\right]^{-1} .
\end{aligned}
$$

The Laplace transform of the kernels [Eq. (C2)] gives

$$
\begin{aligned}
\tilde{k}_{I}^{k \rightarrow j}(\lambda) & =\frac{1}{2} C_{k j} C_{k j} \tilde{G}^{k \rightarrow j}(\lambda), \\
\tilde{k}_{R}^{k \rightarrow j}(\lambda) & =C_{k j} C_{k j} k_{R}^{k \rightarrow j}(\lambda)\left(\tilde{G}^{k \rightarrow j}\right)^{2}(\lambda) .
\end{aligned}
$$

Now we use the fact that $F_{j \rightarrow i}=\sum_{k} \tilde{F}_{k \rightarrow j}$ as well as $k_{I}=$ $(n-1) \tilde{k}_{I}$ and $k_{R}=(n-1) \tilde{k}_{R}$, and we assume that all the couplings are the same. Therefore,

$$
\begin{aligned}
& k_{I}(\lambda)=(n-1) \tilde{k}_{I}(\lambda)=(n-1) \frac{1}{2} C^{2} \tilde{G}(\lambda), \\
& k_{R}(\lambda)=(n-1) \tilde{k}_{R}(\lambda)=(n-1) C^{2} k_{R} \tilde{G}^{2}(\lambda),
\end{aligned}
$$

Subsequently we use definition of $G(\lambda)$ to solve Eq. (C5) for $k_{I}(\lambda)$ and find

$$
\begin{aligned}
k_{I}(\lambda) & =(n-1) \frac{1}{2} C^{2} \tilde{G}_{0}(\lambda)\left[1-\tilde{G}_{0}(\lambda) k_{I}^{k \rightarrow j}(\lambda)\right]^{-1} \\
& =\frac{\tilde{G}_{0}^{-1}(\lambda)}{2}\left[1 \pm \sqrt{1-2(n-1) C^{2} \tilde{G}_{0}^{2}(\lambda)}\right]
\end{aligned}
$$

From Eq. (B19) we know that $\tilde{G}_{0}(\lambda)=\frac{2}{m} \frac{1}{\lambda^{2}+\omega^{2}}$. It is clear we should take the negative sign in front of the square root, as otherwise the Laplace transform does not decay with parameter $\lambda$ at infinity. To derive the actual message as a function of time we follow the definition of $k_{I}^{k \rightarrow j}(\lambda)$,

$$
\begin{aligned}
k_{I}(\lambda)= & 2(n-1) \frac{C^{2}}{m} \\
& \times \frac{1}{\left(\omega^{2}+\lambda^{2}\right)+\sqrt{\left(\omega^{2}+\lambda^{2}\right)^{2}-\left(\frac{2}{m}\right)^{2} 2(n-1) C^{2}}} \\
= & \frac{m}{4} a^{4} \frac{1}{\left(\omega^{2}+\lambda^{2}\right)+\sqrt{\left(\lambda^{2}+\omega_{1}^{2}\right)\left(\lambda^{2}+\omega_{2}^{2}\right)}},
\end{aligned}
$$

where $a^{4}=\left(\frac{8(n-1)}{m^{2}}\right) C^{2}$, and $\omega_{1}^{2}=\omega^{2}-a^{2}$ and $\omega_{2}^{2}=\omega^{2}+a^{2}$.

Equation $(\mathrm{C} 8)$ can be rewritten in a convenient form:

$$
\begin{aligned}
k_{I}(\lambda)= & \frac{m}{4}\left\{\frac{a^{4}}{\sqrt{\left(\lambda^{2}+\omega_{1}^{2}\right)\left(\lambda^{2}+\omega_{2}^{2}\right)}}\right. \\
& \left.-\left[\frac{\left(\lambda^{2}+\omega^{2}\right)^{2}}{\sqrt{\left(\lambda^{2}+\omega_{1}^{2}\right)\left(\lambda^{2}+\omega_{2}^{2}\right)}}-\left(\lambda^{2}+\omega^{2}\right)\right]\right\} .
\end{aligned}
$$

The inverse Laplace transform of the first term is [48]

$$
\begin{aligned}
\mathcal{L}^{-1} & {\left[\frac{1}{\sqrt{\left(\lambda^{2}+\omega_{1}^{2}\right)\left(\lambda^{2}+\omega_{2}^{2}\right)}}\right] } \\
& =\int_{0}^{t} d \tau J_{0}\left(\omega_{1} \tau\right) J_{0}\left(\omega_{2}(t-\tau)\right) \equiv f(t) .
\end{aligned}
$$

To compute the inverse transform of the terms in bracket we exploit the property of the Laplace transform $\mathcal{L}\left[f^{(n)}(t)\right]=$ $\lambda^{n} F(\lambda)-\sum_{k=1}^{n} \lambda^{n-k} f^{k-1}(0)$, where $f^{k}(0)$ denotes $k$ th derivative of $f(t)$ calculated $t=0$. In the case considered here we have $\mathcal{L}^{-1}\left[\lambda^{4} F(\lambda)\right]=f^{(4)}(t)+\mathcal{L}^{-1}\left[\sum_{k=1}^{4} \lambda^{4-k} f^{(k-1)}(0)\right]$, and $\quad \mathcal{L}^{-1}\left[\lambda^{2} F(\lambda)\right]=f^{(2)}(t)+\mathcal{L}^{-1}\left[\sum_{k=1}^{2} \lambda^{2-k} f^{k-1}(0)\right]$, where $F(\lambda)=\frac{1}{\sqrt{\left(\lambda^{2}+\omega_{1}^{2}\right)\left(\lambda^{2}+\omega_{2}^{2}\right)}}$. Taking those relations into account one arrives at the expression for the imaginary kernel in the time domain

$$
\begin{aligned}
k_{I}(\tau) & =\mathcal{L}^{-1}\left[k_{I}(\lambda)\right] \\
& =\frac{m}{4}\left\{a^{4} f(\tau)-\left[f^{(4)}(\tau)+2 \omega^{2} f^{(2)}(\tau)+\omega^{4} f(\tau)\right]\right\},
\end{aligned}
$$

where in the derivation we use the fact that $f(0)=0$, $f^{(1)}(0)=1, f^{(2)}(0)=0, f^{(3)}(0)=-\omega^{2}, f^{(4)}(0)=0$. In this way we have reduced the inverse Laplace transform to a convolution of Bessel functions and derived combinations thereof.

\section{Second version of the calculation}

For an alternative version of the calculation it is convenient to restate the iteration of the Vernon transform in the Laplace domain as

$$
\tilde{k}_{I}^{n+1}(\lambda)=\frac{(n-1) C^{2}}{2} \tilde{G}_{0}(\lambda)\left[1-\tilde{G}_{0}(\lambda) \tilde{k}_{I}^{n}(\lambda)\right]^{-1},
$$

where as above $\tilde{G}_{0}(\lambda)=\frac{2}{m} \frac{1}{\lambda^{2}+\omega^{2}}$ is twice the Laplace transform of the harmonic oscillator response function. 


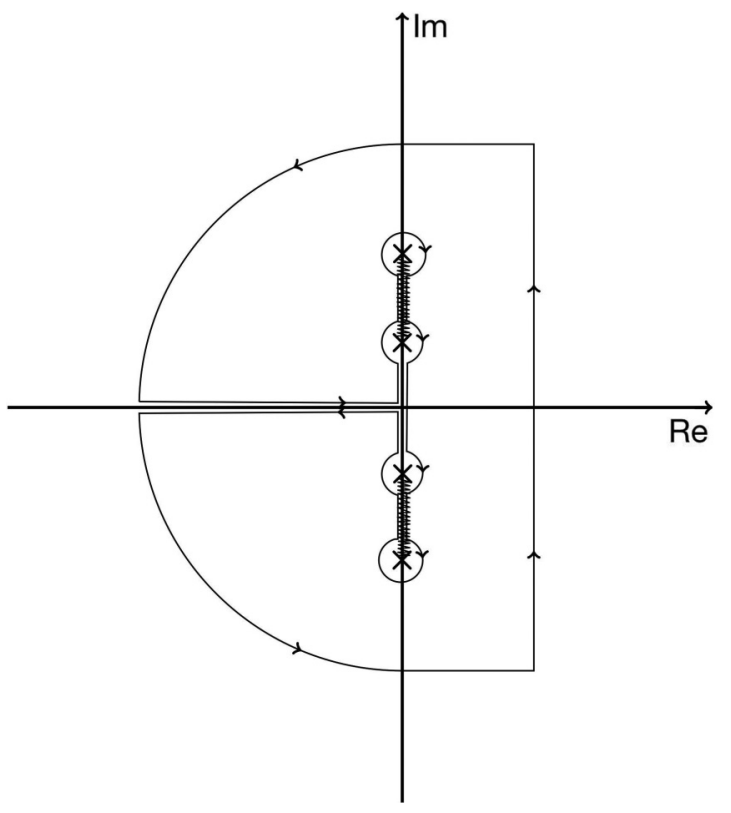

FIG. 4. Illustration of the computation of the inverse Laplace transform of the fixed point kernel from Eq. (C13). By definition this is the integral over a vertical path far enough to the right in the complex plane. Moving the path to the left part of the complex plane one picks up branch cut contributions determined in the text. as

The fixed point can thus be written [equivalent to Eq. (C8)]

$$
\tilde{k}_{I}^{*}(\lambda)=m \frac{\lambda^{2}+\omega^{2}}{4}\left(1-\sqrt{1-\frac{8(n-1) C^{2}}{m^{2}\left(\lambda^{2}+\omega^{2}\right)^{2}}}\right) .
$$

The fixed point kernel in the time domain is given by an inverse Laplace transform:

$$
k_{I}(\tau)=\frac{1}{2 \pi i} \int \tilde{k}_{I}(\lambda) e^{\lambda \tau} d \lambda
$$

The integral is to be performed on a vertical contour far enough to the right in the complex plane. Since the integrand goes down as $\lambda^{-2}$ for large $\lambda$ such an integral has a finite value. If the contour can be moved to the far left in the complex plane, then that integral will be zero because $e^{\lambda \tau}$ then acts as dampening. The inverse Laplace transform is hence given by the integrals encircling poles and cut-lines encountered when moving the integral contour as illustrated in Fig. 4.

The kernel $\tilde{k}_{I}(\lambda)$ is analytic everywhere except in the neighborhood of points where the argument of the square root vanishes. These points are at

$$
i \lambda_{ \pm \pm}= \pm i \sqrt{\omega^{2} \pm \sqrt{8(n-1)} C / m} .
$$

The arguments of the outer square root in above is positive, hence the four points all lie on the imaginary axis. The kernel $\tilde{k}_{I}(\lambda)$ is analytic around the real line as well as for large enough $\lambda$. The contour therefore needs to encircle two cuts between respectively $i\left(\lambda_{+-}, \lambda_{++}\right)$and $i\left(\lambda_{--}, \lambda_{-+}\right)=$ $i\left(-\lambda_{++},-\lambda_{+-}\right)$.

It is convenient to rewrite the square root in Eq. (C12) as $\sqrt{\left(\lambda^{2}+\lambda_{+-}^{2}\right)\left(\lambda^{2}+\lambda_{++}^{2}\right)}$. On the imaginary axis the argument of the square root is then positive for $z<\lambda_{+-}$, negative in the interval $\left(\lambda_{+-}, \lambda_{++}\right)$, and positive again for $z>\lambda_{++}$. The phase of the square root is zero on the imaginary axis up to just below the start of the cut at $i \lambda_{+-}$. Along the cut and just to the right the absolute value of the square root is $\sqrt{\left(z^{2}-\lambda_{+-}^{2}\right)\left(-z^{2}+\lambda_{++}^{2}\right)}$ and the phase is $i$. At the same point along the cut and just to the left the phase is $-i$. The value of the integral encircling $\left(\lambda_{+-}, \lambda_{++}\right)$in the counterclockwise direction is hence

$$
\begin{aligned}
& (+) \text {-side } \\
& \quad=\int_{\lambda_{+-}}^{\lambda_{++}} e^{i z \tau}(2 i)\left(-\frac{1}{2}\right) \sqrt{\left(z^{2}-\lambda_{+-}^{2}\right)\left(-z^{2}+\lambda_{++}^{2}\right)}, d(i z),
\end{aligned}
$$

where $-\frac{1}{2}$ is the prefactor of the square root in Eq. (C12).

For the integral encircling $\left(\lambda_{--}, \lambda_{-+}\right)$one can start from that the phase of the square root must be zero on the imaginary axis just above the cut. Along the cut and just to the left the phase is $+i$, and to the right it is $-i$. The value of this integral, encircling this cut in the positive direction, is thus

$$
\begin{aligned}
(-) \text {-side }= & \int_{-\lambda_{++}}^{-\lambda_{+-}} e^{i z \tau}(-2 i)\left(-\frac{1}{2}\right) \\
& \times \sqrt{\left(z^{2}-\lambda_{+-}^{2}\right)\left(-z^{2}+\lambda_{++}^{2}\right)}, d(i z) \\
= & \int_{\lambda_{+-}}^{\lambda_{++}} e^{-i z \tau}(-2 i)\left(-\frac{1}{2}\right) \\
& \times \sqrt{\left(z^{2}-\lambda_{+-}^{2}\right)\left(-z^{2}+\lambda_{++}^{2}\right)}, d(i z) .
\end{aligned}
$$

Combining both integrals and bringing out a dimensional factors we have a rather simple integral representation

$$
k_{I}(\tau)=\Lambda \int_{q}^{1} \sin \left(\lambda_{++} x \tau\right) \sqrt{\left(x^{2}-q^{2}\right)\left(1-x^{2}\right)} d x,
$$

where $\Lambda=m \lambda_{++}^{3} / \pi$, and where we have used $q=\frac{\lambda_{+-}}{\lambda_{++}}$. The expression in Eq. (C17) is analogous to the Mehler-Sonine representation of the Bessel function $J_{0}(\omega t)$, which is in fact nothing but the inverse Laplace transform of the function $\frac{1}{\sqrt{\Lambda^{2}+\omega^{2}}}$.

The representation Eq. (C17) lends itself to a physical interpretation as follows. The total Hamiltonian in the tree subtended from $j$ will have normal modes. The kernel of the real Feynman-Vernon action on $i$ is according to the general formula

$$
k_{I}(\tau)=\int d \omega \sin \omega \tau J(\omega) d \omega,
$$

where $J(\omega)$ is the spectral density. Comparing Eqs. (C17) and (C18) we have the nontrivial result

$$
J(\omega)= \begin{cases}\sqrt{\left(\omega^{2}-\lambda_{+-}^{2}\right)\left(\lambda_{++}^{2}-\omega^{2}\right)} & \text { for } \omega \in\left[\lambda_{++}, \lambda_{+-}\right], \\ 0 & \text { otherwise. }\end{cases}
$$

In other words, the infinite network as to its influence on one system, behaves for this ferromagnetic harmonic oscillator example as a bath with compact spectral support. 


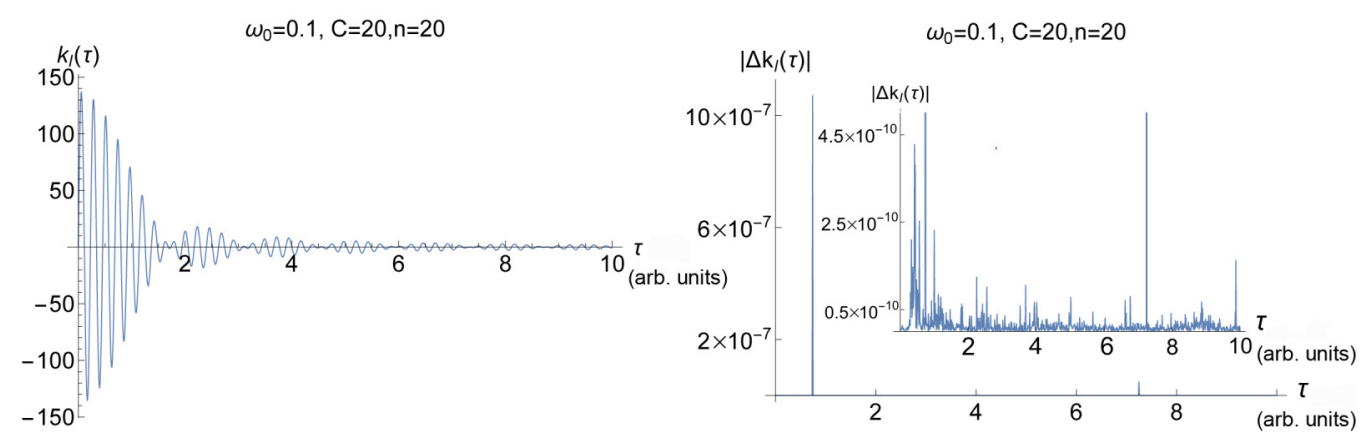

FIG. 5. Numerical comparison of the two formulas for the inverse Laplace transform of the imaginary kernel Eqs. (C13) and (C17). In the left panel the two formulas are plotted together. In the right panel absolute value of the difference between the two formulas (note a different scale in the inset). The parameters used to compute the kernels are The parameters are: $\omega_{0}=0.1, C=20, n=20, m=\frac{1}{2}$. Extensive numerical comparison of the two formulas for different set of parameters allows us to conclude that they are the same.

The Fourier transform of the function $k_{I}(\tau)$ in Eq. (C18), when the value is zero for negative $\tau$, is the Laplace transform of $k_{I}(\tau)$ of argument $1 v$. Since $\hat{k}_{I}(-v)=\left[\hat{k}_{I}(v)\right]^{*}$ it is enough to consider positive $v$. The function $k_{I}(i v)$ is hence real except on the cutline (on positive imaginary $\lambda$ axis), where it is

$$
\hat{k}_{I}^{*}(v)=m \frac{-v^{2}+\omega^{2}}{4}\left[1-i \sqrt{\frac{4(n-1) C^{2}}{m^{2}\left(-v^{2}+\omega^{2}\right)^{2}}-1}\right] .
$$

The Fourier transform of a real function satisfies $\hat{k}_{I}^{*}(-v)=$ $\left[\hat{k}_{I}^{*}(v)\right]^{*}$. We note for further reference that on the cutlines we have

$$
\begin{aligned}
\hat{k}_{I}^{*}(v) \hat{k}_{I}^{*}(-v) & =\left(m \frac{-v^{2}+\omega^{2}}{4}\right)^{2} \frac{8(n-1) C^{2}}{m^{2}\left(-v^{2}+\omega^{2}\right)^{2}} \\
& =\frac{(n-1) C^{2}}{2} .
\end{aligned}
$$

We further note that the cavity kernels $\hat{k}_{I}^{*}(v)$ are of the Belief Propagation $n$-type messages (variables to interactions).

The fixed point kernel $k_{I}^{*}$ in the time domain is illustrated in Fig. 2 and as to the difference of the two ways of computing it in Fig. 5.

\section{APPENDIX D: STABILITY ANALYSIS OF THE DETERMINISTIC REPLICA SYMMETRIC SOLUTION FOR FERROMAGNETIC MODEL ON $\boldsymbol{n}$-REGULAR RANDOM GRAPHS}

In this Appendix we consider the stability of the fixed point found in the main body of the paper, in the context of Replica Symmetry. We hence here consider parameters such that the fixed point exists. The starting point is then that $k$ and $\tilde{k}$ in that analysis are not values but arguments of probability distributions that satisfy compatibility conditions. The goal is to check whether Dirac $\delta$ distributions are stable solutions of these compatibility conditions. We thus start from the BP update equations for Feynman-Vernon kernels in Laplace transform picture written as

$$
Q(k)=\int \prod_{k}^{n-1} d \hat{k}_{j} P\left(\hat{k}_{j}\right) \delta\left(k-\sum_{j} \hat{k}_{j}\right)
$$

and

$$
P(\hat{k})=\int d k Q(k) \delta\left[\hat{k}-f_{\lambda}(k)\right]
$$

where $f_{\lambda}(k)=\frac{C^{2}}{2} \frac{G_{0}(\lambda)}{1-G_{0}(\lambda) k}$ to be expanded around $k=k_{0}=$ $(n-1) k^{*}$ is the Vernon transform applied to Laplace transform variable with parameter $\lambda . G_{0}(\lambda)=\frac{3}{m} \frac{1}{\lambda^{2}+\omega^{2}}$ is twice the response function of the free harmonic oscillator.

Instead of taking $P$ and $Q \delta$ functions we then assume $P(\hat{k}) \sim e^{-\frac{1}{2 \sigma^{2}}\left(\hat{k}-k^{*}\right)^{2}}$ That from Eq. (D1) leads directly to

$$
Q(k) \sim e^{-\frac{1}{2 \sigma^{2}(n-1)}\left[k-(n-1) k^{*}\right]^{2}} .
$$

The point now is to check whether this is consistent with a new Gaussian $P(\hat{k})$. This is clearly not the case for general $f_{\lambda}(k)$. Therefore, a reasonable approach is to check if for $\sigma$ small enough, the variance of $P(\hat{k})$ grows or goes to zero. In the second case, we say that the deterministic solution $k^{*}$ in Eqs. (D1) and (D2) is stable. Otherwise, it is not.

We then proceed to estimate $\left\langle\hat{k}^{\alpha}\right\rangle$, which in practice translates into solving the following integral:

$$
\begin{aligned}
\left\langle\hat{k}^{\alpha}\right\rangle & =\int d \hat{k}^{\alpha} \int d k Q(k) \delta\left[\hat{k}-f_{\lambda}(k)\right] \\
& =\int d k Q(k) f_{\lambda}(k)^{\alpha}=\int d k e^{-\frac{1}{2 \sigma^{2}(n-1)}\left[k-(n-1) k^{*}\right]^{2}} f_{\lambda}(k)^{\alpha} .
\end{aligned}
$$

We expand the function $f_{\lambda}(k)^{\alpha}=\left[\frac{C^{2}}{2} \frac{G_{0}(\lambda)}{1-G_{0}(\lambda) k}\right]^{\alpha}$ around $k=$ $k_{0}=(n-1) k^{*}$. For the expected value $(\alpha=1)$ we have

$$
\begin{gathered}
\frac{G_{0}(\lambda)}{1-G_{0}(\lambda) k}=\frac{G_{0}(\lambda)}{1-G_{0}(\lambda) k_{0}}+\left[\frac{G_{0}(\lambda)}{1-G_{0}(\lambda) k_{0}}\right]^{2}\left(k-k_{0}\right) \\
+\left[\frac{G_{0}(\lambda)}{1-G_{0}(\lambda) k_{0}}\right]^{3}\left(k-k_{0}\right)^{2},
\end{gathered}
$$

such that

$$
\begin{aligned}
\langle k\rangle= & \frac{C^{2}}{2}\left\{\frac{G_{0}(\lambda)}{1-G_{0}(\lambda) k_{0}} \int d k e^{-\frac{1}{2 \sigma^{2}(n-1)}\left(k-k_{0}\right)^{2}}\right. \\
& \left.+\left[\frac{G_{0}(\lambda)}{1-G_{0}(\lambda) k_{0}}\right]^{3} \int d k e^{-\frac{1}{2 \sigma^{2}(n-1)}\left(k-k_{0}\right)^{2}}\left(k-k_{0}\right)^{2}\right\} \\
= & \frac{C^{2}}{2} \frac{G_{0}(\lambda)}{1-G_{0}(\lambda) k_{0}}\left\{1+\sigma^{2}(n-1)\left[\frac{G_{0}(\lambda)}{1-G_{0}(\lambda) k_{0}}\right]^{2}\right\} .
\end{aligned}
$$


Similarly, for the second moment $(\alpha=2)$

$$
\begin{aligned}
& {\left[\frac{G_{0}(\lambda)}{1-G_{0}(\lambda) k}\right]^{2}} \\
& \quad=\left[\frac{G_{0}(\lambda)}{1-G_{0}(\lambda) k_{0}}\right]^{2}+2\left[\frac{G_{0}(\lambda)}{1-G_{0}(\lambda) k_{0}}\right]^{3}\left(k-k_{0}\right) \\
& \quad+\frac{6}{2}\left[\frac{G_{0}(\lambda)}{1-G_{0}(\lambda) k_{0}}\right]^{4}\left(k-k_{0}\right)^{2}
\end{aligned}
$$

and

$$
\begin{aligned}
\left\langle k^{2}\right\rangle= & \frac{C^{4}}{4}\left[\frac{G_{0}(\lambda)}{1-G_{0}(\lambda) k_{0}}\right]^{2}\{1 \\
& \left.+3 \sigma^{2}(n-1)\left[\frac{G_{0}(\lambda)}{1-G_{0}(\lambda) k_{0}}\right]^{2}\right\} .
\end{aligned}
$$

Putting everything together we have

$$
\begin{aligned}
\left\langle k^{2}\right\rangle-\langle k\rangle^{2}= & \sigma^{2} \frac{(n-1)}{4} \\
& \times C^{4}\left[\frac{G_{0}(\lambda)}{1-G_{0}(\lambda) k_{0}}\right]^{4} .
\end{aligned}
$$

The variance of the distribution after passing through the BP update is hence proportional to original variance $\sigma^{2}$. Note that the combination $C \frac{G_{0}(\lambda)}{1-G_{0}(\lambda) k_{0}}$ is dimensionless, and that $\sigma$ has the same dimension as $k$.

Since we expand around the fixed point $k^{*}$ we have $k_{0}=(n-1) k^{*}, \frac{G_{0}(\lambda)}{1-G_{0}(\lambda) k_{0}}=\frac{2}{C^{2}(n-1)} k^{*}$, and $k^{*}=m \frac{\lambda^{2}+\omega^{2}}{4}[1-$ $\sqrt{1-\frac{8(n-1) C^{2}}{m^{2}\left(\lambda^{2}+\omega^{2}\right)^{2}}}$. For small $C$ we can neglect the difference between $\omega$ and $\omega_{0}$ and have

$$
k^{*} \approx \frac{(n-1) C^{2}}{m\left(\lambda^{2}+\omega_{0}^{2}\right)} \quad(C \text { small })
$$

and hence

$$
\frac{G_{0}(\lambda)}{1-G_{0}(\lambda) k_{0}} \approx \frac{1}{\left(\lambda^{2}+\omega_{0}^{2}\right)} \quad(C \text { small })
$$

For small values of $C$ the proportionality is hence less than one, and by iteration the Gaussian gets sharper. This means that the deterministic ( $\delta$ function) solution is stable.

\section{APPENDIX E: REPLICA ANALYSIS OF OSCILLATOR FREQUENCY DISORDER}

In this Appendix we give the details of the analyze the effects of disorder in frequencies. As we will eventually be mostly interested in the effects on $k_{R}$ it will be convenient to consider the iteration of the cavity equation for $k_{I}$ in the Fourier domain. Our starting point will hence be the following mappings of $n-1$ complex numbers $z_{1}, z_{2}, \ldots, z_{n-1}$ on one complex number $z^{\prime}$ :

$$
z^{\prime}=\mathcal{V}\left(z_{1}, z_{2}, \ldots ; \omega, C, n\right)=a \frac{1}{b-\sum_{k} z_{k}} .
$$

The variables $z$ represents $\hat{k}_{I}(v)$ (interaction-to-variable message of at Fourier component $v$ ) and the parameters as defined in the main body of the paper and above are

$$
\begin{gathered}
a=\frac{C^{2}}{2}, \\
b=\frac{m}{2}\left(-v^{2}+\omega^{2}\right), \\
\omega^{2}=\omega_{0}^{2}+\frac{n C}{m} .
\end{gathered}
$$

To this we now add

$$
\omega_{0}=\bar{\omega}+\Delta \omega
$$

where $\bar{\omega}$ is a baseline bare oscillator frequency and $\Delta \omega$ is quenched random variable of mean zero and variance $\overline{(\Delta \omega)^{2}}$. We note the $\Delta \omega=0$ fixed point

$$
z^{\prime}=z_{1}=z_{2}=\ldots=z_{n-1}=p
$$

given by

$$
p=\frac{b}{2(n-1)} \pm \sqrt{\frac{b^{2}}{4(n-1)^{2}}-\frac{a}{(n-1)}} .
$$

The expression inside the square root is positive if $a<\frac{b^{2}}{4(n-1)}$ or $\left(-v^{2}+\bar{\omega}^{2}\right)>\frac{C}{m} \sqrt{8(n-1)}$. This is always possible when $v$ is sufficiently different from $\bar{\omega}$. The fixed point Fourier $\tilde{k}_{I}(v)$ is then real, and by analytic continuation from the Laplace transform we should take the negative sign of the square root. However, the expression inside the square root is negative if $a>\frac{b^{2}}{4(n-1)}$. This happens in a band of frequencies $\mathcal{I}$ sufficiently close to $\bar{\omega}$ given by

$$
\mathcal{I}_{\bar{\omega}, m, C, n}=\left[\sqrt{\bar{\omega}^{2}-\frac{C}{m} \sqrt{8(n-1)}}, \sqrt{\bar{\omega}^{2}+\frac{C}{m} \sqrt{8(n-1)}}\right]
$$

and the reflected interval for negative frequencies. By analytic continuation we then have

$$
\begin{aligned}
& z^{*}(v)=\frac{b}{2(n-1)}+i \sqrt{\frac{a}{n-1}-\frac{b^{2}}{4(n-1)^{2}}}, \\
& z^{*}(-v)=\frac{b}{2(n-1)}-i \sqrt{\frac{a}{n-1}-\frac{b^{2}}{4(n-1)^{2}}} .
\end{aligned}
$$

We note the quantity entering in the cavity equation for $k_{R}(v)$ :

$$
z^{*}(v) z^{*}(-v)=|p|^{2}=\frac{a}{(n-1)}=\frac{C^{2}}{2(n-1)} .
$$

In the band the multiplier of the cavity equation for $k_{R}(v)$ is independent of $v$, combined with the prefactor $\frac{4}{C^{2}}(n-1)$ discussed above and in the main text, it equals 2 . In $\mathcal{I}_{\bar{\omega}, m, C, n}$. It is therefore convenient to write

$$
p=C[2(n-1)]^{-\frac{1}{2}} e^{i \phi},
$$

where the phase $\phi \in[0, \pi]$ parametrizes the frequency $v$ and the fixed point $p$ in the band. For the following discussion it is further convenient to introduce the abbreviation

$$
\sum_{k=1}^{n-1}=(n-1) p+\Delta z
$$


and note the following derivatives of $\mathcal{V}$ at $\Delta z=\Delta \omega=0$ in $\mathcal{I}_{\bar{\omega}, m, C, n}$ :

$$
\begin{gathered}
\frac{d \mathcal{V}}{d \Delta z}=\mathcal{V}^{\prime}[(n-1) p]=\frac{2}{C^{2}} p^{2}=\frac{1}{n-1} e^{2 i \phi}, \\
\frac{d^{2} \mathcal{V}}{d(\Delta z)^{2}}=\mathcal{V}^{\prime \prime}[(n-1) p]=2\left(\frac{2}{C^{2}}\right)^{2} p^{3} \\
=\sqrt{8(n-1)} \frac{1}{C(n-1)^{2}} e^{3 i \phi}, \\
\frac{d \mathcal{V}}{d \Delta \omega}=-\frac{2 m \bar{\omega}}{C^{2}} p^{2}=-\frac{m \bar{\omega}}{n-1} e^{2 i \phi}, \\
\frac{d^{2} \mathcal{V}}{d \Delta \omega d \Delta z}=-2 \frac{2 m \bar{\omega}}{C^{2}} \frac{2}{C^{2}} p^{3}=\sqrt{8(n-1)} \frac{m \bar{\omega}}{C(n-1)^{2}} e^{3 i \phi} \\
\frac{d^{2} \mathcal{V}}{d(\Delta \omega)^{2}}=-\frac{2 m}{C^{2}} p^{2}+2\left(\frac{2 m \bar{\omega}}{C^{2}}\right)^{2} p^{3} \\
=-\frac{m}{n-1} e^{2 i \phi}+\sqrt{8(n-1)} \frac{m^{2} \bar{\omega}^{2}}{C(n-1)^{2}} e^{3 i \phi}
\end{gathered}
$$

\section{Small-disorder RS calculation}

The procedure here is similar to the small-disorder expansion for the Anderson model on a tree carried out by Miller and Derrida [44]. We chose to not represent messages of type $k_{I}$ (variable-to-interaction BP messages). We therefore do not make explicit the " $n$-type" update Eq. (D1) but incorporate it in the " $m$-type" update Eq. (D2). The latter however must now also take into account the disorder in frequencies which leads to a probability distribution on the real and imaginary parts of the Fourier component $k_{I}(v)$. The equation to consider is hence

$$
\begin{aligned}
P^{\prime}\left(\operatorname{Re} z^{\prime}, \operatorname{Im} z^{\prime}\right)= & \mathbf{E}_{\Delta \omega} \int \delta\left\{\operatorname{Re}\left[z^{\prime}\right]-\operatorname{Re}\left[\mathcal{V}\left(z_{1}, \ldots, z_{n-1}\right)\right]\right\} \\
& \times \delta\left\{\operatorname{Im}\left[z^{\prime}\right]-\operatorname{Im}\left[\mathcal{V}\left(z_{1}, \ldots, z_{n-1}\right)\right]\right\} \\
& \left.\prod_{k=1}^{n-1} P(\operatorname{Ee} 19), \operatorname{Re} z_{k}, \operatorname{Im} z_{k}\right) d \operatorname{Re} z_{k} d \operatorname{Im} z_{k} . \quad(\mathrm{E} 20)
\end{aligned}
$$

The two-dimensional measure $d \operatorname{Re} z d \operatorname{Im} z$ can be abbreviated $d z \wedge d z^{*}$ and the two-dimensional distribution $P(\operatorname{Re} z, \operatorname{Im} z)$ can be written $P\left(z, z^{*}\right)$. A Gaussian distribution on the real and imaginary parts of a complex number $z$ is characterized by the first and second moments

$$
\begin{aligned}
\bar{z} & =\int z P\left(z, z^{*}\right) d z \wedge d z^{*}, \\
\overline{z^{2}} & =\int z^{2} P\left(z, z^{*}\right) d z \wedge d z^{*}, \\
\overline{|z|^{2}} & =\int|z|^{2} P\left(z, z^{*}\right) d z \wedge d z^{*} .
\end{aligned}
$$

Note that $\overline{z^{*}}=(\bar{z})^{*}$ and $\overline{\left(z^{*}\right)^{2}}=\left(\overline{z^{2}}\right)^{*}$. Assuming Gaussian closure we need to approximate the same moments for the distribution $P^{\prime}$, which gives

$$
\begin{gathered}
\overline{z^{\prime}}=\mathbf{E}_{\Delta \omega} \int \mathcal{V}\left(z_{1}, \ldots, z_{n-1}\right) \prod_{k=1}^{n-1} P\left(z_{k}, z_{k}^{*}\right) d z_{k} \wedge d z_{k}^{*} \\
\overline{\left(z^{\prime}\right)^{2}}=\mathbf{E}_{\Delta \omega} \int \mathcal{V}^{2}\left(z_{1}, \ldots, z_{n-1}\right) \prod_{k=1}^{n-1} P\left(z_{k}, z_{k}^{*}\right) d z_{k} \wedge d z_{k}^{*} \\
\overline{\left|z^{\prime}\right|^{2}}= \\
\mathbf{E}_{\Delta \omega} \int \mathcal{V}\left(z_{1}, \ldots, z_{n-1}\right) \mathcal{V}^{*}\left(z_{1}, \ldots, z_{n-1}\right) \\
\times \prod_{k=1}^{n-1} P\left(z_{k}, z_{k}^{*}\right) d z_{k} \wedge d z_{k}^{*}
\end{gathered}
$$

The above equations are then evaluated in Taylor expansion around $p$ and $p^{*}$, which gives

$$
\begin{aligned}
\overline{z^{\prime}}=p+\frac{d \mathcal{V}}{d \Delta z}(n-1) \overline{z-p}+\frac{1}{2} \frac{d^{2} \mathcal{V}}{d(\Delta z)^{2}}\left[(n-1) \overline{(z-\bar{z})^{2}}\right. \\
\left.+(n-1)^{2}(\overline{z-p})^{2}\right]+\frac{1}{2} \frac{d^{2} \mathcal{V}}{d(\Delta \omega)^{2}} \overline{(\Delta \omega)^{2}} \\
\overline{\left(z^{\prime}\right)^{2}}-\left(\overline{z^{\prime}}\right)^{2}=\left\{\mathcal{V}^{\prime}[(n-1) p]\right\}^{2}(n-1)\left[\overline{z^{2}}-(\bar{z})^{2}\right] \\
+\left(\frac{d \mathcal{V}}{d \Delta \omega}\right)^{2} \overline{(\Delta \omega)^{2}} \\
\overline{\left|z^{\prime}\right|^{2}}-\left|\overline{z^{\prime}}\right|^{2}=\left|\mathcal{V}^{\prime}[(n-1) p]\right|^{2}(n-1)\left(\overline{|z|^{2}}-\left|\bar{z}^{\prime}\right|^{2}\right) \\
+\left|\frac{d \mathcal{V}}{d \Delta \omega}\right|^{2} \overline{(\Delta \omega)^{2}} .
\end{aligned}
$$

For $n=2$ (a line) the last two equations are not relaxations. In particular, $\overline{\left(z^{\prime}\right)^{2}}-\left(\overline{z^{\prime}}\right)^{2}$ has resonant contributions from the two endpoints of the band and the middle, at the points where $e^{4 i \phi}=1$. This means that the Gaussian ansatz is not stable under iteration. For $n>2$ (a tree) the last two equations are, however, relaxations, and give

$$
\begin{gathered}
\overline{(z)^{2}}-(\bar{z})^{2}=\left(1-\frac{1}{n-1} e^{4 i \phi}\right)^{-1}\left(\frac{m \bar{\omega}}{n-1}\right)^{2} e^{4 i \phi} \overline{(\Delta \omega)^{2}}, \\
\overline{|z|^{2}}-|\bar{z}|^{2}=\left(1-\frac{1}{n-1}\right)^{-1}\left(\frac{m \bar{\omega}}{n-1}\right)^{2} \overline{(\Delta \omega)^{2}} .
\end{gathered}
$$

The iteration equation for $\overline{z-p}$ is not a relaxation for any $n$, but a perturbed rotation. Substituting in the relaxed quantities it reads

$$
\begin{aligned}
\overline{z^{\prime}-p}= & e^{2 i \phi} \overline{(z-p)}+\frac{\sqrt{2(n-1)}}{C} e^{3 i \phi} \overline{(z-p)^{2}} \\
& \times \overline{(\Delta \omega)^{2}}\left(\frac{1}{2} \frac{m}{n-1} e^{2 i \phi}+(m \omega)^{2} \frac{2}{C} e^{3 i \phi}\right.
\end{aligned}
$$




$$
\begin{aligned}
& +\left(1-\frac{1}{n-1} e^{4 i \phi}\right)^{-1}\left(\frac{m \bar{\omega}}{n-1}\right)^{2} e^{4 i \phi} \\
& \left.\times \frac{2}{C}\left(\frac{1}{n-1}\right)^{\frac{3}{2}} e^{3 i \phi}\right)+ \text { h.o.t. }
\end{aligned}
$$

Assuming that only resonant terms matter the above can be simplified to

$$
\overline{z^{\prime}-p} \approx e^{2 i \phi}\left[\overline{z-p}+\frac{1}{2} \frac{m}{n-1} \overline{(\Delta \omega)^{2}}\right] .
$$

The assumption is not valid for small $C$. This is so because the interval where the derivative changes phase from zero to $\pi$ is of width proportional to $C$; hence, for small $C$ the complex derivatives are large. However, for moderately large $C$ and small enough $\overline{(\Delta \omega)^{2}}$ it should be justified. Nevertheless, Eq. (E33) describes a rotation and not a relaxation which reaches a fixed point. Hence, the Gaussian ansatz is also not stable for a tree.

\section{RS analysis of the rotating distribution}

By a slight extension of the argument we can analyze also the rotating distribution. As the new starting point we assume the distribution on each incoming link to be characterized by $\overline{z_{k}-p}$ which is a small complex number. We assume the phase of this complex number to be uniformly randomly distributed over $[0,2 \pi]$. The goal is to deduce from the this a stationary distribution over the absolute values of $|\overline{z-p}|$ and eventually $|z|^{2}$, the quantity which matters in the iteration equation for $k_{R}(v)$. Instead of Eq. (E33) we hence have

$$
\overline{z^{\prime}-p} \approx e^{2 i \phi}\left[\frac{1}{n-1} \sum_{k=1}^{n-1} \overline{z_{k}-p}+\frac{1}{2} \frac{m}{n-1} \overline{(\Delta \omega)^{2}}\right]
$$

For the absolute value squared we have

$$
\begin{aligned}
\left|\overline{z^{\prime}-p}\right|^{2} \approx & {\left[\frac{1}{2} \frac{m}{n-1} \overline{(\Delta \omega)^{2}}\right]^{2} } \\
& +\frac{1}{(n-1)^{2}}\left[\sum_{k}\left|\overline{z_{k}-p}\right|^{2}+\sum_{k \neq l} \overline{z_{k}-p}\left(\overline{z_{l}-p}\right)^{*}\right]
\end{aligned}
$$

Assuming all incoming distribution equally distributed in radius and uniformly random in phase and taking the expectation value with respect to this metadistribution we find

$$
\mathbf{E}\left[|\overline{z-p}|^{2}\right] \approx \frac{m^{2}}{4(n-1)(n-2)}\left[\overline{(\Delta \omega)^{2}}\right]^{2} \equiv r .
$$

Note that we have already assumed $n>2$ (a tree); for $n=2$ (a line) the above equation is not valid and there is not a stable solution for the metadistribution. For the tree we can however evaluate quantities such as

$$
\mathbf{E}\left[|z|^{2}\right] \approx \frac{1}{2 \pi} \int_{0}^{2 \pi}\left|p+r e^{i \theta}\right|^{2} d \theta=|p|^{2}+r^{2} .
$$

Since $\frac{4}{C^{2}}(n-1)|p|^{2}=2$ for all frequencies in the band $\mathcal{I}$, small disorder in the oscillator frequencies does not change the conclusion that the real fix point $k_{R}(t, v)$ is exponentially large in the duration of the process (in $t-t_{i}$ ). Hence, such systems will eventually turn classical.

\section{Remarks on the strong disorder case}

In this Appendix we supplement the remarks in the main body of the paper on the case of strong disorder. It is convenient to also assume that while disorder large compared to the interaction, it is also small compared to the bare oscillator frequency. When this is the case we have

$$
\frac{C}{m} \ll \overline{(\Delta \omega)^{2}} \ll \bar{\omega}^{2},
$$

where $\bar{\omega}$ is the expected value of the bare oscillator frequency. The interval Eq. (E8) then reads

$$
\mathcal{I} \approx\left[\bar{\omega}-\frac{C}{m \bar{\omega}} \sqrt{2(n-1)}, \bar{\omega}+\frac{C}{m \bar{\omega}} \sqrt{2(n-1)}\right] .
$$

The assumption in the strong disorder case is that the width of this interval is much less than $\sigma_{\omega}=\sqrt{\overline{(\Delta \omega)^{2}}}$ which follows from the first inequality in Eq. (E38). The variable $k_{I}(v)$ has the same dimension as $C$. We can therefore introduce the dimensionless frequency shift as $v=\bar{\omega}+\tilde{v} \frac{C}{m \bar{\omega}}$, a dimensionless oscillator frequency disorder parameter as $\Delta \omega=s \sigma_{\omega}$ and the dimensionless mapping

$$
\tilde{\mathcal{V}}(x+i y)=\frac{1}{C} \mathcal{V}[(x+i y) C]=\frac{1}{2} \frac{1}{A s-\tilde{v}-x-i y} .
$$

In the above $A=\frac{\bar{\omega} \sigma_{\omega}}{C / m}$ is a dimensionless number much larger than one, and we have neglected terms from $v^{2}$ and $\Delta \omega^{2}$. Successive iterations of $\mathcal{V}$ will then be characterized by different values of the random parameter $s$, which by the above have the same effect as large fluctuations in the dimensionless frequency $\tilde{v}$. Supposing now that a fixed point distribution of such a process exits we are to consider $k_{R}(v)$ after many applications of the cavity equation, which means multiplying many factors $\frac{4}{C^{2}}|z|^{2}$. In the band without disorder this factor is 2 , but outside the band it decays to zero. To know if such a product typically increases or decreases we should estimate the expectation value of $\log \frac{4}{C^{2}}|z|^{2}$. When the disorder in the oscillator frequencies is larger than the width of the band the distribution is sampled often where $\frac{4}{C^{2}}|z|^{2}$ is less than one. Contrary to the previous limit for large enough disorder in the oscillator frequencies $k_{R}(v)$ therefore eventually tends to zero for all $v$. In this limit the quantum network hence behaves as a dissipative but still decoherence-free environment.
[1] M. Mézard and A. Montanari, Information, Physics and Computation (Oxford University Press, Oxford, UK, 2009).
[2] T. Richardson and R. Urbanke, Modern Coding Theory (Cambridge University Press, Cambridge, UK, 2008). 
[3] M. J. Wainwright and M. I. Jordan, FNT Mach. Learn. 1, 1 (2008).

[4] J. S. Yedidia, W. T. Freeman, and Y. Weiss, Understanding Belief Propagation and Its Generalizations (Morgan Kaufmann Publishers, San Francisco, CA, 2003), pp. 239-269.

[5] R. Abou-Chacra, D. J. Thouless, and P. W. Anderson, J. Phys. C: Solid State Phys. 6, 1734 (1973).

[6] M. Dupont, N. Laflorencie, and G. Lemarié, Phys. Rev. B 102, 174205 (2020).

[7] G. Parisi, S. Pascazio, F. Pietracaprina, V. Ros, and A. Scardicchio, J. Phys. A: Math. Theor. 53, 014003 (2020).

[8] I. García-Mata, J. Martin, R. Dubertrand, O. Giraud, B. Georgeot, and G. Lemarié, Phys. Rev. Research 2, 012020(R) (2020).

[9] C. Laumann, A. Scardicchio, and S. L. Sondhi, Phys. Rev. B 78, 134424 (2008).

[10] V. Bapst, L. Foini, F. Krzakala, G. Semerjian, and F. Zamponi, Phys. Rep. 523, 127 (2013).

[11] M. B. Hastings, Phys. Rev. B 76, 201102(R) (2007).

[12] M. Leifer and D. Poulin, Ann. Phys. 323, 1899 (2008).

[13] D. Poulin and E. Bilgin, Phys. Rev. A 77, 052318 (2008).

[14] L. B. Ioffe and M. Mézard, Phys. Rev. Lett. 105, 037001 (2010).

[15] O. Dimitrova and M. Mézard, J. Stat. Mech.: Theory Exp. (2011) P01020.

[16] H. Loeliger and P. O. Vontobel, IEEE Trans. Inf. Theory 63, 5642 (2017).

[17] J. M. Renes, New J. Phys. 19, 072001 (2017).

[18] R. P. Feynman and J. Vernon, Ann. Phys. 24, 118 (1963).

[19] Hitting a quantum volume chord: IBM quantum adds six new systems with quantum volume 32 , https://www.ibm.com/blogs/ research/2020/07/qv32-performance/.

[20] S. Perseguers, M. Lewenstein, A. Acín, and J. I. Cirac, Nat. Phys. 6, 539 (2010).

[21] R. Beals, S. Brierley, O. Gray, A. W. Harrow, S. Kutin, N. Linden, D. Shepherd, and M. Stather, Proc. R. Soc. A 469, 20120686 (2013).

[22] S. Boixo, S. V. Isakov, V. N. Smelyanskiy, R. Babbush, N. Ding, Z. Jiang, M. J. Bremner, J. M. Martinis, and H. Neven, Nat. Phys. 14, 595 (2018).

[23] A. W. Cross, L. S. Bishop, S. Sheldon, P. D. Nation, and J. M. Gambetta, Phys. Rev. A 100, 032328 (2019).

[24] Y. Nam and D. Maslov, npj Quantum Inf. 5, 44 (2019).

[25] Y. Song, M. Kim, H. Hwang, W. Lee, and J. Ahn, Phys. Rev. Research 3, 013286 (2021).

[26] J. Preskill, Quantum 2, 79 (2018).

[27] B. M. Terhal, Rev. Mod. Phys. 87, 307 (2015).
[28] B. Derrida, E. Gardner, and A. Zippelius, Europhys. Lett. 4, 167 (1987).

[29] I. Neri and D. Bollé, J. Stat. Mech.: Theory Exp. (2009) P08009.

[30] E. Aurell and H. Mahmoudi, J. Stat. Mech.: Theory Exp. (2011) P04014.

[31] E. Aurell and H. Mahmoudi, Phys. Rev. E 85, 031119 (2012).

[32] G. Del Ferraro and E. Aurell, Phys. Rev. E 92, 010102(R) (2015).

[33] T. Barthel, C. De Bacco, and S. Franz, Phys. Rev. E 97, 010104(R) (2018).

[34] T. Barthel, J. Stat. Mech.: Theory Exp. (2020) 013217.

[35] E. Aurell, G. Del Ferraro, E. Domínguez, and R. Mulet, Phys. Rev. E 95, 052119 (2017).

[36] E. Aurell, E. Domínguez, D. Machado, and R. Mulet, Phys. Rev. E 97, 050103(R) (2018).

[37] E. Aurell, E. Domínguez, D. Machado, and R. Mulet, Phys. Rev. Lett. 123, 230602 (2019).

[38] E. Domínguez, D. Machado, and R. Mulet, J. Stat. Mech.: Theory Exp. (2020) 073304.

[39] D. Machado and R. Mulet, Phys. Rev. E 104, 054303 (2021).

[40] E. Aurell, R. Kawai, and K. Goyal, J. Phys. A: Math. Theor. 53, 275303 (2020).

[41] F. L. Vernon, Ph.D. thesis, California Institute of Technology, 1959, https://resolver.caltech.edu/CaltechETD:etd-02242006154616.

[42] A. E. Rubio López, C. Gonzalez-Ballestero, and O. RomeroIsart, Phys. Rev. B 98, 155405 (2018).

[43] A. Crisanti, G. Paladin, and A. Vulpiani, Products of Random Matrices in Statistical Physics, Vol. 104 (Springer-Verlag, Berlin, 1993).

[44] J. D. Miller and B. Derrida, J. Stat. Phys. 75, 357 (1994).

[45] E. B. Sudderth, A. T. Ihler, W. T. Freeman, and A. S. Willsky, in Proceedings of the IEEE Computer Society Conference on Computer Vision and Pattern Recognition, Madison, WI, USA (IEEE, Piscataway, NJ, 2003), Vol. 1, p. I.

[46] D. Bickson, A. T. Ihler, H. Avissar, and D. Dolev, in Proceedings of the 47th Annual Allerton Conference on Communication, Control, and Computing (Allerton), Monticello, IL, USA (IEEE, Piscataway, NJ, 2009), pp. 439-446.

[47] E. Aurell and J. Tuziemski, arXiv:2103.13255 (2021).

[48] In Table of Integrals, Series, and Products (8th Edition), edited by D. Zwillinger, V. Moll, I. Gradshteyn, and I. Ryzhik (Academic Press, Boston, MA, 2014), pp. 1077-1103, https://www.sciencedirect.com/science/article/pii/ B9780123849335000126. 